\title{
1 Comprehensive Topographical Map of the Serotonergic Fibers in the Mouse Brain
}

2 Janak R. Awasthi ${ }^{1,2}$, Kota Tamada ${ }^{1}$, Eric T. N. Overton ${ }^{1}$, Toru Takumi ${ }^{1,2,3^{*}}$

$3 \quad{ }^{1}$ RIKEN Brain Science Institute, Wako, Saitama 351-0198, Japan

$4 \quad{ }^{2}$ Graduate School of Science and Engineering, Saitama University, Sakura, Saitama 338-8570,

5 Japan

$6{ }^{3}$ Department of Physiology and Cell Biology, Kobe University School of Medicine, Chuo,

7 Kobe 650-0017, Japan

8

9 *Correspondence to:

10 Toru Takumi

11 RIKEN Center for Brain Science, 2-1 Hirosawa, Wako, Saitama 351-0198, Japan.

12 TEL: +8148467 5906, FAX: +8148467 6079

13 Email: toru.takumi@riken.jp

15 Funding information

16 This work was supported partly by KAKENHI (16H06316, 16H06463) from Japan Society for

17 the Promotion of Science and Ministry of Education, Culture, Sports, Science, and Technology,

18 Intramural Research Grant for Neurological and Psychiatric Disorders of NCNP, the Takeda

19 Science Foundation and Smoking Research Foundation. Awasthi has been awarded the

20 International Program Associate (IPA) fellowship from the RIKEN - Saitama University joint

21 frontier program, Japan. 


\section{Abstract}

24 It is well established that serotonergic fibers distribute throughout the brain. Abnormal 25 densities or patterns of serotonergic fibers have been implicated in neuropsychiatric disorders.

26 Although many classical studies have examined the distribution pattern of serotonergic fibers,

27 most of them were either limited to specific brain areas or had limitations in demonstrating the 28 fine axonal morphology. In this study, we utilize transgenic mice expressing GFP under the 29 SERT promoter to map the topography of serotonergic fibers across the rostro-caudal extent of each brain area. We demonstrate previously unreported regional density and fine-grained anatomy of serotonergic fibers. Our findings include: 1) SERT fibers distribute abundantly in the thalamic nuclei close to the midline and dorsolateral areas, in most of the hypothalamic nuclei with few exceptions such as the median eminence and arcuate nuclei, and within the basal amygdaloid complex and lateral septal nuclei, 2) the source fibers of innervation of the hippocampus traverse through the septal nuclei before reaching its destination, 3) unique, filamentous type of straight terminal fibers within the nucleus accumbens, 4) laminar pattern of innervation in the hippocampus, olfactory bulb and cortex with heterogenicity in innervation density among the layers, 5) cortical labelling density gradually decreases rostro-caudally, 6) fibers traverse and distribute mostly within the gray matter, leaving the white fiber bundles uninnervated, and 7) most of the highly labelled nuclei and cortical areas have predominant anatomical connection to limbic structures. In conclusion, we provide novel, regionally specific

42 insights on the distribution map of serotonergic fibers using transgenic mouse.

43 Keywords: Serotonin, SERT, transgenic mouse, whole brain mapping 


\section{INTRODUCTION}

Serotonin (5-hydroxytryptamine or 5-HT) is a well-recognized modulator of brain activity and other functions in peripheral organs (Berger et al., 2009). Dysfunction of 5-HT system has been implicated in mood disorders, schizophrenia, addiction, attention deficit hyperactivity disorder, autism spectrum disorders (ASD) and other mental disorders (Lin et al., 2014). In fact, results of several biological studies demonstrate alterations of morphology and/or density of 5-HT neurons in several mental disorders such as depression (Rajkowska et al., 2017), ASD (Makkonen et al., 2008) (Tamada and Takumi, 2015), schizophrenia (Hrovatin et al., 2019), traumatic brain injury (Abe et al., 2016) and in cases like prenatal exposure of psychotropic drugs (Xu et al., 2004) (Maciag et al., 2006) (Weaver et al., 2010), neonatal hypoxia (Reinebrant et al., 2020), or postnatal social isolation (Kuramochi and Nakamura, 2009).

The 5-HT fibers originate from discretely organized cell somas in the raphe nuclei of brainstem and project throughout the brain (Fuxe, 1965) (Vertes and Linley, 2008). In parallel, the activities of these raphe cell bodies are regulated by the monosynaptic inputs from the forebrain and brainstem neurons (Pollak Dorocic et al., 2014). A total of 28,000 5-HT producing neurons in the mouse brain (Ishimura et al., 1988) does not act as a single population, but rather contains parallel sub-systems that differ in connectivity, physiological properties, and behavioral functions (Ren et al., 2018) (Okaty et al., 2019). Its diversity is additionally exhibited by recent transcriptomics study suggesting that the dorsal raphe (DR) 5-HT neurons co-expressing Vglut-3 preferentially innervate the cortex, whereas those co-expressing thyrotropin-releasing hormones innervate the subcortical regions (Ren et al., 2019). The anterograde tracing approaches have revealed that serotonergic efferents from different raphe nuclei to the major brain regions are distinctive and largely non-overlapping (Muzerelle et al., 2016). Although these recent dissections of serotonergic system have unearthed its much finer 
details, the precise and comprehensive topographical map of total 5-HT efferents in whole brain remains undetermined by the recent neuroanatomical tools.

The serotonergic topography has long been studied with the progressive advent of neuroanatomical techniques such as aldehyde histofluorescence (Fuxe, 1965), autoradiography (Parent et al., 1981), immunohistochemical techniques using antibodies against 5-HT marker enzyme tryptophan hydroxylase (Tph) (Pickel et al., 1977) or 5-HT itself (Steinbusch, 1981). However, they were accompanied by several limitations: These techniques neither allowed the unambiguous identification of labelled 5-HT or non-5-HT neurons, nor were they ideal for visualizing the fine terminal axons due to either the dilution of tracer in highly ramified axons (Parent et al., 1981) or rapid metabolization of released 5-HT, requiring pretreatment before tissue harvesting (Azmitia and Gannon, 1983). Moreover, the synthesis rate or metabolism of 5-HT or Tph, which is subjected to large variability depending on the environmental conditions, may contribute to the alteration in the staining outcome (Nielsen et al., 2006). Therefore, the actual picture of 5-HT innervation in the brain must be more extensive than that revealed by these techniques.

To ensure high fidelity visualization of the fine-grain anatomy of the serotonergic system, we employed SERT (serotonin transporter) -EGFP (enhanced green fluorescence protein) bacterial artificial chromosome (BAC) transgenic mouse in which an EGFP reporter gene is inserted upstream of the coding sequence of the SERT gene (Schmidt et al., 2013). Combined with GFP antibody and high-resolution imaging, we present better organization of serotonergic fibers with the complete visualization of the whole axons, their morphological features and distribution across the whole brain of the adult mice.

\section{MATERIAL AND METHODS}

\subsection{Animals}


92 All procedures for animal experiments were carried out in accordance with the guidelines of

93 the animal experimentation committee of RIKEN, Japan. The mouse strain used for this research was STOCK Tg (Slc6a4-EGFP) JP55Gsat/Mmucd (RRID: MMRRC_030692-UCD) which was obtained from Research Resources Division, RIKEN. Originally, the mice were obtained from the Mutant Mouse Resource and Research Center (MMRRC) at University of California at Davis, a NIH-funded strain repository, and was donated to the MMRRC by Nathaniel Heintz, The Rockefeller University (Gong et al., 2003). The mice line was maintained by mating male SERT-EGFP mice with wild type female C57BL/6J mice (backcrossed more than 5 times). All mice were maintained on a 12/12-hour light/dark cycle and housed in standard plexiglass cages with food and water ad libitum.

\subsection{Immunohistochemistry}

103 Eleven-week-old male mice $(n=4)$ were anesthetized and transcardially perfused with

104 phosphate buffer saline (PBS) followed by 4\% paraformaldehyde (PFA) in $0.1 \mathrm{M}$ phosphate buffer (pH 7.4). Brains were removed and immersion-fixed with $4 \%$ PFA at $4{ }^{\circ} \mathrm{C}$ overnight.

106 Coronal $(100 \mu \mathrm{m}$ thick) and sagittal (60 $\mu \mathrm{m}$ thick) sections were made using Leica VT1200 S

107 vibratome (Leica Microsystems, Nussloch, Germany) and collected in PBS containing 0.01\% sodium azide and stored at $4{ }^{\circ} \mathrm{C}$ until use. Immunohistochemical procedures were performed on free floating sections. Sections were treated with $0.3 \% \mathrm{H}_{2} \mathrm{O}_{2}$ in PBS for 30 minutes, washed with PBS for 5 minutes three times, incubated with a blocking solution (PBS containing 3\%

111 normal goat serum and $0.3 \%$ Triton X-100) for 30 minutes at room temperature, and incubated

112 with primary antibodies [Anti GFP rabbit polyclonal antibody (1:500 dilution; \#catalog no: A-

113 11122, Thermo Fisher Scientific, Tokyo, Japan)] diluted with the blocking solution overnight

114 at $4^{\circ} \mathrm{C}$. Sections were washed with PBS containing 0.3\% Triton X-100 (PBST) for 10 minutes

115 four times, incubated with fluorescent-conjugated secondary antibodies [Alexa Fluor 488 Goat 116 anti-Rabbit IgG (1:500 dilution; catalog \# A-11034; Thermo Fisher Scientific, Tokyo, Japan)] 
117 diluted with the blocking solution for 2 hours at room temperature, washed with PBST for 10

118 minutes four times, transferred onto Superfrost slides, and mounted with VECTASHIELD with

119 DAPI (Vector Laboratories, Burlingame, CA, USA)

\section{$120 \quad 2.3$ Images acquisition and analysis}

121 The sections were imaged at 10X magnification with VS120 (Olympus, Tokyo, Japan) whole

122 slide scanner fluorescent microscope and FV3000 confocal microscope (Olympus, Tokyo,

123 Japan). The images were processed using ImageJ (Rasband, 1997) and Adobe photoshop CS6,

124 and were converted into grayscale and adjusted for brightness and contrast. Finally, the borders

125 were manually drawn around the nuclei using Inkscape software (Harrington, 2005) upon

126 comparing the SERT immunolabelled sections with its DAPI stained counterparts with the aid

127 of the Allen brain atlas (Allen Institute for Brain Science, 2004). The density of serotonergic

128 fibers in each nucleus was then rated on a scale of 0 to 6 (Table 1).

129 Table 1 Fiber density ratings

\begin{tabular}{lll}
\hline Density rating & Description & Definition \\
\hline 0 & Scanty & lowest density of fibers; more background than fibers \\
1 & Sparse & low, more background than fibers \\
2 & Mild & slightly more background than fibers \\
3 & Moderate & balance between fibers and background \\
4 & Moderately high & more fibers than background \\
5 & Dense & possible to distinguish individual fibers \\
6 & Very dense & highest density of fibers; difficult to distinguish individual fibers \\
\hline
\end{tabular}

130 The representative image for each scale is depicted in supplementary figure 1.

\section{3. RESULTS}

132 In this study, we comprehensively mapped the distribution pattern of the serotonergic fibers

133 across the rostro-caudal extent of each brain regions and sub-regions using the SERT-EGFP

134 transgenic mice that was genetically modified to express GFP in the serotonergic fibers. The

135 serotonergic fibers arising from the discrete clusters of cell bodies in the midbrain raphe nuclei

136 innervated nearly all the brain areas in a specific pattern and innervation density. The study 
137 plan and the results are depicted schematically in supplementary figure 2 . In the following

138 account, we describe the projection pattern and rostro-caudal density gradient in each nucleus

139 of various brain areas and their sub-divisions.

\subsection{INNERVATION PATTERN IN THE THALAMUS}

141 We analyzed the innervation density and the pattern of SERT-EGFP fibers across the rostro-

142 caudal extent of different thalamic nuclei. No thalamic nuclei were devoid of the innervation.

143 In order to ease the description, we categorized the thalamic nuclei according to the recent

144 classification concept viz; (1) midline and intralaminar nuclei (2) association nuclei (3)

145 principal nuclei (4) epithalamus and (5) the reticular nucleus (Groenewegen and Witter, 2004).

146 Towards the rostral pole of the thalamus, we observed that some of the dorsally diverted

147 collaterals of pathway fibers from the lateral preoptic area (LPO) and lateral hypothalamic area

148 (LHA) passed around the column of fornix and/or along the zona incerta (ZI) to project into

149 the ventrally located thalamic nuclei such as the nucleus reuniens (RE) (Figure $1 \mathrm{~B} \& 2 \mathrm{C}$,

150 arrow). Meanwhile, towards the caudal thalamic levels, we noticed that laterally directed

151 collaterals of pathway fibers passed across the ZI to innervate the lateral geniculate (LG) nuclei

152 (Figure 1G). Similarly, rostrally directed collaterals of pathway fibers from the periaqueductal gray (PAG) (Figure 1G), which traversed through the paraventricular thalamic nuclei (PVT)

154 (Figure 1E, arrow), provided the innervation to the dorsally located thalamic nuclei of caudal pole (ex; PVT and other neighboring nuclei).

\section{$156 \quad$ 3.1.1 Midline nuclei}

157 The midline nuclei include paraventricular thalamic (PVT), paratenial (PT), rhomboid (RH) nuclei and nucleus reuniens (RE). We observed that the entire anteroposterior axis of these nuclei was very densely $(6+)$ labeled with a couple of exceptions. For example, the labeling

160 density within RH nuclei decreased to moderate (3+) density at its caudal extent (Figure 1E),

161 while the PT nucleus was densely (5+) labeled (Figure 1A). We found that the ascending 
pathway fibers traversing ahead from PAG were clustered as two bilateral bundles within the caudal PVT (Figure 1E, arrow) which diffused rostrally to innervate the other adjacent nuclei.

164 There were other nuclei such as the intermediodorsal (IMD), interanteromedial (IAM), and 165 interanterodorsal (IAD), which were located in the midline but were not considered part of the 166 midline nuclei.

\section{$167 \quad 3.1 .2$ Intralaminar nuclei}

168 These nuclei were anatomically associated with the medullary lamina of thalamus, thus were considered as a separate category despite their location in the thalamic midline. It consists of

170 central lateral (CL), paracentral (PC), central medial (CM) and parafascicular (PF) nuclei

171 (Groenewegen and Witter, 2004). We observed that SERT-EGFP fiber density within the CM nuclei gradually decreased from very dense $(6+)$ labelling (Figure 1A) to sparse (1+) density

173 (Figure 1D), and progressively became milder (2+) towards its caudal extent (Figure 1F). We also noticed an intranuclear gradient at its caudal extent (Figure 1F). Similarly, we found that labelling density within CL nuclei gradually decreased from dense (5+) (Figure 1C) to sparse

$176(1+)$ (Figure 1E) rostro-caudally. Likewise, innervation density within PC nuclei changed from

177 moderate $(3+)$ (Figure 1C) to scanty (0) level (Figure 1E) along the rostro-caudal extent, whereas PF nuclei exhibited intranuclear gradient with milder (2+) density on its dorsal aspect and sparse $(1+)$ ventrally (Figure $1 \mathrm{~F})$.

\subsubsection{Association nuclei}

181 According to the aforementioned classification, thalamic association nuclei include the anterior

182 nuclei and its subdivisions [anteroventral (AV), anterodorsal (AD), anteromedial (AM), interanteromedial (IAM)], lateral dorsal (LD), lateral posterior (LP), mediodorsal (MD), intermediodorsal (IMD) and submedial thalamic (SMT) nuclei. Among the different subdivisions of the anterior nuclei, we observed that AV nuclei were densely (5+) labelled

186 throughout the rostro-caudal extent (Figure 1A-C). Similarly, AD nuclei received moderately 
187 high (4+) projections (Figure 1A-B). AM and IAD nuclei had milder $(2+)$ projections (Figure

188 1B), whereas the labelling density within IAM nuclei decreased from moderately high (4+)

189 (Figure 1B) to milder (2+) density (Figure 1C) rostro-caudally. We spotted that LD (Figure

190 1B-E) and LP (Figure 1E-F) nuclei were densely (5+) labeled with slight decrease in their

191 labelling density towards their caudal extent. The density of fibers within the MD nuclei

192 decreased from moderate $(3+)$ to mild $(2+)$ one rostro-caudally (Figure 1 C-E). However, it

193 exhibited higher labelling within the central (c) part compared to its medial (m) and lateral (l)

194 area (Figure 1C-D). Similarly, the innervation density within IMD nuclei decreased from dense

$195(5+)$ (Figure 1C) to moderate $(3+)$ labelling (Figure 1F) along the rostro-caudal axis.

196 Interestingly, the fiber density within the submedial nucleus exhibited distinct rostral-caudal

197 gradient being moderately $(3+)$ labeled at the rostral half (Figure 1B), sparsely $(1+)$ at the

198 intermediate level (Figure 1C) and its caudal extent was very densely (6+) labelled (Figure 1D-

199 E).

\section{$200 \quad$ 3.1.4 Principal nuclei}

201 Principal nuclei include the ventrobasal (VB) complex [ventral posteromedial (VPM) and

202 ventral posterolateral nuclei (VPL)], ventroanterior lateral complex (VAL), ventromedial nucleus (VM), posterior nucleus (PO), the lateral (LG) and medial geniculate (MG) nuclei

204 (Groenewegen and Witter, 2004). We noticed that the VB, VAL complexes, VM, and PO nuclei received almost no innervation $(<1)$ (Figure 1D-F) except the PO nucleus at its rostral

206 extent, which was sparsely (1+) labelled (Figure 1D). The LG nuclei complex was very densely

$207(6+)$ innervated throughout the rostro-caudal extent (Figure 1E-G). However, the medial area 208 of the ventral LG nuclei (LGv) had comparably less innervation (Figure 1F). Similarly, we noticed that MG nuclei were mildly (2+) labeled only on its lateral area (Figure $1 \mathrm{G})$, thus

210 showing the intranuclear gradient. We observed that the fascicles of SERT-EGFP fibers arising 
211 from the PAG passed laterally along ZI to enrich the innervation of MG and LG nuclei (Figure

$2121 \mathrm{G})$.

\section{$213 \quad$ 3.1.5 Medial and lateral habenula (epithalamus)}

214 We found that the habenula was less innervated compared to other dorsally located thalamic

215 nuclei. Medial habenula were sparsely (1+) labelled towards the rostral pole (Figure 1B). As

216 traced caudally, the whole area of lateral habenula and only the lateral part of the medial

217 habenula were moderately (3+) labelled (Figure 1C-D). This pattern was due to the collaterals

218 of pathway fibers ascending ahead through the PVT being directed laterally along the borders

219 in-between the medial and lateral habenula. The labelling density within the lateral habenula

220 was moderately high (4+) towards the caudal extent (Figure 1F). Overall, the lateral habenula was innervated in higher density compared to the medial habenula.

\subsubsection{Reticular nucleus}

Except the moderately (3+) labelled rostral pole of reticular nuclei (Figure 1A), the rest of its extension received sparse $(1+)$ innervation (Figure B-F). However, the innervation density was slightly higher than adjacently located ventrobasal complex of the thalamus (Figure 1D).

\subsubsection{Other areas}

227

The white fiber bundles; viz. fasciculus retroflexus (fr), mamillotegmental tract (mmt), the innervation. However, few SERT-EGFP fibers passed around the white fibers within the cerebral peduncle (Figure 1F) to reach the globus pallidus (GP). We observed the collaterals

231 of pathway fibers travelling within the stria medullaris (sm) and stria terminalis (st). Within stria terminalis, the fibers were dispersed throughout the tract (Figure 6D). However, fibers were clustered only in the dorsal part of sm leaving the rest of the part being devoid of the

234 innervation (Figure 1A, arrow head). 
236 We categorized hypothalamic nuclei according to a conventional classification method based

237 on regional location, viz; preoptic region, supraoptic region, tuberal region and mammillary

238 region (D. G. Stuart, 1962). Preoptic and supraoptic regions were located towards the rostral

239 hypothalamic zone, and the tuberal region was situated in the middle hypothalamic zone,

240 whereas the mamillary region was positioned in the caudal hypothalamic zone. We noticed that

241 the hypothalamic nuclei were heterogeneously innervated.

\subsubsection{Preoptic region (Figure 2A-B)}

243 The ascending forebrain bundle traversed rostrally through lateral preoptic region (LPO). Few

244 thin, punctate fibers of innervation were scattered in between the thick ascending pathway

245 fibers. Some of the detached fibers from the main ascending bundle were clustered separately

246 within the substantia innominata (SI). The median preoptic nucleus (MEPO) and rostral part

247 of medial preoptic area (MPO) were densely (5+) innervated (Figure 2A). However, the

248 labelling density in MPO decreased as traced caudally, exhibiting the intranuclear gradient.

249 The fibers were distributed moderately high (4+) on its lateral area and moderately $(3+)$ on its

250 medial part. (Figure 2B). The anteroventral periventricular nuclei (AVPV) were mildly (2+)

251 innervated (Figure 2A), whereas the medial preoptic nucleus (MPN) received moderately high

$252(4+)$ projection (Figure 2B). The other non-principal hypothalamic nuclei present in the preoptic region; viz, magnocellular nuclei (MA) and nucleus of diagonal band (NDB) were densely (5+) innervated (Figure 2A), although the labelling density in MA decreased slightly as traced caudally (Figure 2B). We observed that the collaterals of pathway fibers which were clustered in SI diverted medially to run over the lower border of MA and NDB in the area adjoining to OT, to finally enter into the septal nuclei (Figure 2A, arrow).

\subsubsection{Supraoptic region (Figure 2C-F)}

The ascending forebrain bundle traversed rostrally through the lateral hypothalamic area (LHA) with few interspersed fibers of innervation. The collaterals of ascending bundle passed 
261 dorsally into the thalamic RE (Figure 2C, arrow) and laterally into the ZI (Figure 2F). Some

262 of the collaterals ran ventromedially through supraoptic commissure and retrochiasmatic nuclei

263 (RCN) (Figure 2F, arrow) to project into the rostrally located suprachiasmatic nuclei (SCN)

264 (Figure 2D). We found that the supraoptic nuclei (SO) (Figure 2C \& F) and rostral part of RCN

265 (Figure 2C) were moderately (3+) labelled. However, the labelling density of latter increased

266 densely $(5+)$ caudally (Figure $2 \mathrm{~F})$. Anterior hypothalamic nuclei (AHN) were densely $(5+)$

267 innervated throughout the rostro-caudal extent. Interestingly, we noticed that SCN exhibited

268 both intranuclear and rostro-caudal gradients. Rostral (Figure 2C) and caudal (Figure 2E) SCN

269 was sparsely (1+) innervated. However, the intranuclear gradient was observed in midway SCN

270 (Figure 2D). Its ventromedial area (core) received very dense (6+) projections making it one

271 of the most heavily innervated brain areas while the dorsolateral area (shell) was sparsely (1+)

272 innervated. Similarly, we observed rostro-caudal gradient in the sub-paraventricular zone

273 (SBPV) in which innervation density increased from moderate $(3+)$ (Figure 2C) to dense $(5+)$

274 levels (Figure 2F). Paraventricular hypothalamic nuclei (PVH) exhibited both intranuclear and

275 rostro-caudal gradients. Rostrally, except its densely (5+) innervated periventricular part

276 (PVHpv) (Figure 2D), the rest of its area was sparsely (1+) labelled (Figure 2C-E). However,

277 at its caudal extent the whole PVH nucleus was densely (5+) labelled (Figure 2F).

\section{$278 \quad 3.2 .3$ Tuberal region (Figure 2G-H)}

279 The ascending forebrain bundle traversed rostrally through the perifornical LHA of tuberal

280 region. The ventromedial hypothalamic nuclei (VMH) exhibited intranuclear gradient across

281 the rostro-caudal axis. Rostrally, its dorsomedial (dm) part was mildly $(2+)$ innervated

282 compared to the rest of the nucleus $(5+)$ (Figure 2G). However, the fibers were densely (5+)

283 and homogeneously distributed throughout the nucleus as traced caudally (Figure $2 \mathrm{H}$ ).

284 Similarly, we noticed that the dorsomedial hypothalamic nuclei (DMH) were densely $(5+)$

285 innervated rostrally (Figure $2 \mathrm{G}$ ) and labelled mildly (2+) at its caudal extent (Figure 2H), 
286 displaying the rostro-caudal gradient. The arcuate nucleus exhibited sparse labelling (1+)

287 throughout the antero-posterior axis. We found that the collateral fibers arising from the main

288 ascending bundle in LHA projected ventrally into the tuberal (TU) nuclei making it densely

$289(5+)$ innervated.

$290 \quad$ 3.2.4 Mamillary region (Figure 2I-K)

291 We observed that the innervation density within the posterior hypothalamic nuclei $(\mathrm{PH})$

292 decreased from dense (5+) (Figure 2H-I) to sparse (1+) density (Figure 2K) rostro-caudally.

293 Moreover, we noticed that PH nuclei served as the route for the collateral fibers (arising from

294 the main ascending bundle in the LHA) to pass dorsally into the thalamus (Figure $2 \mathrm{H}$ ).

295 Similarly, the posterior part of periventricular nuclei (PVp) (Figure 2I-J) and supramamillary

296 nuclei (SuM) (Figure 2J, 2K) had sparse (1+) labelling throughout the rostro-caudal extent.

297 Likewise, the both ventral and dorsal parts of premamillary (PMv, PMd) nuclei were labelled

298 in moderately high (4+) density (Figure 2I). We spotted collaterals arising from the ascending

299 fiber bundle at VTA running directly into the lateral mamillary nuclei (LM) making it densely

300 (5+) innervated (Figure 2J, arrow). Similarly, the ventral part of tuberomammillary (TMv) was

301 labelled in moderately high (4+) density (Figure 2J), whereas the dorsal part being mildly (2+)

302 innervated (Figure 2I). The median part of medial mamillary nuclei (MMme) was densely (5+)

303 labelled, while the rest had innervation with slightly less density (4+) (Figure 2J). However,

304 the pattern was in contrary to its caudal extent (Figure 2K). Strikingly, the median eminence

305 had almost no SERT-EGFP labelled fibers (Figure 2H-I).

306 There were few nuclei which extended over more than one region and the patterns

307 they exhibited were as follows: the mediolaterally extended nuclei ZI carried the collaterals of

308 the main ascending bundle from LHA towards laterally located thalamic reticular nuclei or the

309 geniculate nuclei (Figure 1E-G). We observed that the SERT-EGFP fiber distributed over ZI

310 gradually decreased from the dense $(5+)$ (Figure $2 \mathrm{~F})$ to sparse $(1+)$ density (Figure 2I) rostro- 
311 caudally. Subthalamic nuclei (STN) that were located just above the cerebral peduncle (cpd)

312 were densely (5+) labelled because of the directly entering collaterals of ascending bundle

313 fibers from LHA (Figure 2H-I). We noticed heterogenicity among the different subdivisions of

314 periventricular nuclei (PV). The preoptic (PVpo) (Figure 2B), anterior (PVa) (Figure 2C), and

315 intermediate (PVi) (Figure 2D) parts of PV nuclei were densely (5+) innervated. The AVPV

316 and PVp nuclei with less innervation have been already included in the preoptic region and

317 mamillary region, respectively.

$318 \quad 3.2 .5$ Other areas within the hypothalamic zone

319 The optic tract (opt) was completely devoid of innervation (Figure 2F-G). However, some

320 fibers which entered into the SCN received serotonergic innervation (Figure 2D, arrow head).

321 The white fiber bundles, viz. fornix (fx) (Figure 2B), mamillotegmental tract (mtt) (Figure 2H-

I), anterior commissure (aco, act) (Figure 2A, B) and cerebral peduncle (cpd) (Figure 2 I-J)

\subsection{INNERVATION PATTERN IN THE AMYGDALA}

325 We observed that no nuclei within the amygdaloid complex were devoid of the serotonergic

326 innervation. However, they exhibited heterogenicity in the innervation density and gradient

327 along the rostro-caudal axis. We noticed that collaterals of pathway fibers entered into the

328 amygdala via two routes, via the lateral hypothalamic area (LHA) / substantia innominata (SI)

329 (Figure 3A, arrow) and via the stria terminalis (st) (Figure 3C-D). The fibers contained in stria

330 terminalis originated from the main ascending bundle at lateral preoptic area (LPO) (Figure

$3316 \mathrm{D}$ ) and ran caudally over the thalamus to ultimately project to the amygdalar nuclei (Figure

$3323 \mathrm{C}, \mathrm{D})$. These collaterals of pathway fibers were scattered among the thin and punctate fibers

333 of innervation and were difficult to trace further. We classified the amygdaloid nuclei into three

334 groups: (1) the deep or basolateral group (2) the superficial or cortical-like group and (3) the 
335 centromedial group (McDonald, 1998). There were other nuclei which did not easily

336 incorporate into any of these groups, which we describe separately.

\section{$337 \quad$ 3.3.1 Deep or basolateral group}

338 According to the aforementioned classification, the basolateral group includes the lateral

339 amygdalar nucleus (LA), basolateral amygdalar nucleus (BLA), and basomedial amygdalar

340 nucleus (BMA). LA nuclei were moderately $(3+)$ labelled throughout the rostro-caudal extent,

341 though the density in its apical portion was less compared to the basal area (Figure 3C-E). We

342 noticed that BLA nuclei were among the most heavily innervated nuclei (Figure 3A-F). Among

343 its different subdivisions, both the anterior (BLAa) and posterior (BLAp) nuclei were very

344 densely (6+) labelled. However, the fiber density within BLAa decreased slightly at its caudal

345 extent. The ventral part (BLAv) was densely (5+) innervated (Figure 3D-E). Similarly, we

346 observed variations in the innervation density among the different subdivisions of BMA nuclei.

347 Its anterior part (BMAa) was densely (5+) labelled (figure 3A-B). However, the innervation

348 density in the posterior part (BMAp) increased from moderate $(3+)$ (Figure 3C) to dense $(5+)$

349 (Figure 3E) level and then decreased again towards its caudal extent (Figure 3F).

$350 \quad$ 3.3.2 Superficial or cortical-like group

351 Superficial or cortical-like group of amygdalar nuclei is also known as corticomedial nuclei. It consists of nucleus of lateral olfactory tract (NLOT), bed nucleus of accessory olfactory tract (BA), anterior and posterior cortical amygdalar nucleus (CoAa and CoAp), and the piriformamygdaloid area (PAA). We noticed that NLOT exhibited change in both the innervation pattern and density along the rostro-caudal axis. It was densely (5+) innervated at its rostral pole without a laminar pattern (fig not shown). At its midway along the rostro-caudal axis, it appeared distinct because of slightly less innervation (4+) compared to its surrounding area (Figure 3A). We observed a tri-laminar pattern at its caudal extent with a less innervated intervening layer (layer 2) compared to the rest of the layers (4+) (Figure 3B). Similarly, BA 
was moderately $(3+)$ labelled (Figure 3B). We also observed that the innervation density within

361 CoAa decreased from moderately high density $(4+)$ to milder (2+) level (Figure 3A-B),

362 whereas the density within CoAp increased from moderate $(3+)$ (Figure 3C) to dense (5+)

363 (Figure 3F) labelling pattern along the rostro-caudal axis. Similarly, fiber density within the

364 PAA also increased from moderate $(3+)$ to dense $(5+)$ pattern (Figure $3 \mathrm{C}-\mathrm{F})$ rostro-caudally.

\section{$365 \quad$ 3.3.3 Centromedial group}

366 The centromedial group consists of medial (MeA) and central amygdalar (CeA) nuclei. CeA

367 nuclei were moderately (3+) labelled throughout the anteroposterior extent (Figure 3B-D)

368 except at its moderately high (4+) labelled rostral most end (Figure 3A). We observed that

369 medial amygdalar nucleus (MEA) exhibited heterogenicity among its different subdivisions.

370 Its anterodorsal part (MEAad) received very dense $(6+)$ to dense $(5+)$ projection fibers along

371 its rostro-caudal extent (Figure 3A-C). The anteroventral (MEAav) (Figure 3C-D), posteroventral (MEApv) (Figure 3E) and posterodorsal (MEApd) (Figure 3D-E) part were moderately $(3+)$ labelled throughout the rostro-caudal axis. However, the boundaries of these nuclei had higher density fibers compared to the core.

\subsubsection{Other nuclei}

The nuclei that did not fit in the above classification include the anterior amygdalar area (AAA)

377 (Figure 3A), intercalated areas (IA) (Figure 3A-C), and posterior amygdaloid nucleus (PA)

378 (Figure 3F), all of which were heavily innervated (See table 2).

\subsection{INNERVATION PATTERN IN THE SEPTUM}

380 The septum has three major parts and their subdivisions. The major areas are the medial septum

381 (MS), lateral septum (LS), and the nuclei of diagonal band (NDB) (Risold, 2004). We observed 
385 for these fibers to reach above the corpus callosum (CC) (where they clustered to form the

386 supracallosal bundle) (Figure 4A, arrow head) and into dorsal fornix (df) and fimbria (fi)

387 (Figure 7A \& E, arrow). Finally, all these fibers culminated into the hippocampus (Figure 7E).

388 On a close inspection of the septal component, we noticed that few thin and varicosed fibers of

389 innervation were scattered among the thick collaterals of pathway fibers. Moreover, we

390 observed heterogenicity in the innervation density and orientation of fibers among the different

391 nuclei.

$392 \quad$ 3.4.1 The nucleus of diagonal band (NBD) and medial septum (MS)

393 The NDB consists of two limbs, the vertical and horizontal limb (Figure 4C, D). The vertical

394 limb is in continuation with the medial septum (MS) and occupies the medial most position in

395 the rostral half of the septum (Figure 4B-D). Towards the rostral pole, we observed that the

396 medially diverted collaterals of ascending pathway fibers ran dorsally along the lateral border

397 of NDB and via the lateral septum (LSr) to reach above the CC, thus forming the supracallosal

398 bundle (SCB) (Figure 4A). The SCB ran caudally over the CC while providing the innervation

399 to the medial part of cortex en-route which finally turned around the splenium of CC to enter

400 into the hippocampus (Figure 7E). In the slight caudal section, some of the medially diverted

401 collaterals of ascending fiber ran dorsally along lateral border of NDB to continue into the MS

402 which then terminated upon surrounding the septo-hippocampal (SH) nuclei (Figure 4B). We

403 observed that at the midway septal level, majority of the fibers entering into the septum along

404 the lateral border of NDB appeared relatively straight and oriented ventro-dorsally to reach up

405 to the ventral border of CC (Figure 4C-D). They gave off many projections to the lateral septum

406 en-route. A particularly striking feature of NDB and MS of the mid-level septum was the

407 moderately $(3+)$ innervated zone lying close to the midline (Figure 4C-D). This zone consisted

408 of mainly the thin fibers of innervation with very few collaterals of ascending pathway fibers.

409 The overall density and pattern of SERT-EGFP fibers in the MS were similar to that of NDB. 
410 In addition, we observed a strikingly very dense (6+) cluster of fibers in the limiting zone

411 between nucleus accumbens (NAc) and lateral septum (LSr) (Figure 4D, yellow arrow head).

\section{$412 \quad 3.4 .2$ Lateral septal nucleus}

413 The lateral septum (LS) consists of three main subdivisions; the caudal (LSc), rostral (LSr) and

414 ventral (LSv). We noticed heterogeneous distribution of SERT-EGFP fibers among the

415 different subdivisions of the LS. The LSc was the least innervated area of the whole septum. It

416 contained vertically oriented fibers at its border abutting the CC (Figure 4C-F, arrow head).

417 Upon tracing these vertical fibers, we noticed they passed caudally into the dorsal fornix which

418 served as one of the sources of innervation of the hippocampus (Figure 7A). The rest of the

419 area of LSc had randomly oriented fibers in minimal density (1+) (Figure 4D-F). However,

420 LSc was moderately labelled at its rostral pole (Figure 4C). Similarly, we found that LSv was

421 moderately (3+) innervated (Figure 4E-F). However, the collaterals of ascending fiber entering

422 into the septum were arborized heavily on its dorsomedial part (arrow head, Figure 4E). We

423 observed that the LSr was among the densely labelled areas of the whole brain, however, it

424 demonstrated heterogenicity in the labelling density and orientation of fibers along the rostro-

425 caudal axis. It was very densely $(6+)$ innervated in the rostral half (Figure 4A-B), densely (5+)

426 with randomly oriented fibers at the mid-level (Figure 4C-D) and the density decreased even

427 further towards its caudal pole (4+) where the fibers were mediolaterally oriented (Figure 4E).

428 Upon tracing the fibers, we noticed that the collaterals of pathway fibers within the LSr passed

429 caudally into septofimbrial nuclei (SF) (Figure 4F) and finally were projected into the fimbria.

430 The fimbria (fi) served as one of the sources of innervation of hippocampus (Figure 7A \& E).

431 Thus, the medially diverted fibers from the main ascending bundle traversed across the

432 different level of septum to reach the supracallosal bundle, fimbria and dorsal fornix which

433 finally culminated into the hippocampus (Figure 7E).

$434 \quad$ 3.4.3 Other Septal areas 
435 There are other areas which are located within the septum but are not its principal components.

436 We observed that septo-hippocampal (SH) nucleus was sparsely (1+) innervated (Figure 4A-

437 B). However, the collaterals of pathway fibers running dorsally across MS terminated along its

438 outskirts (Figure 4B). Innervation in the insula magna (islm), the largest islands of Calleja,

439 exhibited the rostro-caudal gradient. The rostral $i s l m$ that was almost devoid of the innervation

440 (Figure 4C) received dense (5+) network of fibers at its caudal extent (Figure 4D). Similarly,

441 column of fornix (fx) appeared completely devoid of the innervation except a thin fascicle of

442 fibers that was observed running dorsally in-between two bilateral columns which reached up

443 to the ventral border of CC (Figure 4E). Triangular septal nucleus (TRS) was sparsely (1+)

444 innervated (Figure 4F). However, SF contained mediolaterally oriented collaterals of pathway

445 fibers in moderately high (4+) density (Figure 4F).

\subsection{INNERVATION PATTERN IN THE BASAL GANGLIA}

447 We observed gradual increase in the labelling density of caudo-putamen (CP) rostro-caudally. The mildly (2+) labelled CP towards its rostral extent (Figure 5A) received moderately high

$449(4+)$ projections towards its caudal pole (Figure 5E). We noticed that the collaterals of main ascending bundle clustered in SI were diverted either medially or laterally to provide the innervation to the $\mathrm{CP}$ (Figure 5A-D). The laterally directed fibers passed along external capsule (ec) (Figure 5A, arrow head) and/or turned around the anterior commissure (aco) to innervate the lateral part of CP (Figure 5B), whereas the medially directed collaterals of pathway fibers

454 passed dorsally across BNST around the aco to reach the medial side of CP (Figure 5C, segmented black arrow line). Thus, because of these different routes of innervation, the density of labelling in the $\mathrm{CP}$ was higher either laterally (Figure 5B) or medially (Figure 5C) at different levels along the rostro-caudal extent. In addition, the collaterals of pathway fibers running rostrally through $\mathrm{CP}$ were clustered together at some points which appeared as patches in the coronal section (arrow Figure 5C, red arrow head). We noticed that the areas through which 
thalamocortical fibers were traversing ahead appeared as circular gaps in the coronal sections as they were unlabeled with GFP (Figure 5C, green arrow head). The SERT-EGFP fibers surrounding these gaps gave it a whorl like appearance. In sagittal sections, the collaterals of pathway fibers entering into the CP via external capsule or GP can be distinguished as smooth, straight, large diameter structures which were more evident in the NAc (Figure 5F \& G, red arrow head). The terminal fibers innervating CP were very fine in morphology. The GP received very dense $(6+)$ serotonergic projection which made it clearly distinguishable from the adjacently located striatum (Figure 5D-E). The collaterals of main ascending fibers streamed into GP from lateral hypothalamic area (LHA) through SI (Figure 5E). In addition, we observed that the pattern of SERT-EGFP fibers in GP external segment (GPe) changed from a whorl pattern (Figure 5D) to homogeneous labelling (Figure 5E) rostro-caudally.

\subsection{INNERVATION PATTERN IN THE NUCLEUS ACCUMBENS}

The innervation pattern in the nucleus accumbens (NAc) was unique because of the presence of two different types of terminal fibers. We observed a unique type of fine, non-varicose, relatively straight terminals scattered among the ubiquitous punctate type of fibers of innervation. These unique types of terminal fiber had morphology similar to pathway fibers but were thin in diameter. They appeared as loosely clustered patch in the coronal sections of caudal NAc (Figure 5C, blue arrow head) and can be visualized much better in the sagittal sections (Figure 5F \& G, red arrow head). We noticed that the innervation density in the NAc was higher rostrally (4+) (Figure 5A) than its caudal pole (3+) (Figure 5C). This was because

480 the loosely clustered fibers at the caudal NAc were scattered throughout the nuclei at the rostral pole.

\subsection{INNERVATION PATTERN IN THE BED NUCLEUS OF STRIA TERMINALIS}

483 We observed that collaterals of pathway fibers clustered within substantia innominata (SI) were

484 diverted medially which turned around the anterior commissure (aco) to reach the medial side 
485 of CP; thus, innervating the bed nuclei of stria terminalis (BNST) en-route (Figure 6A-B and

486 5C). However, towards the caudal pole of BNST, the detached fibers from the main ascending

487 bundle at lateral preoptic area (LPO) entered into the BNST which then continued into the stria

488 terminalis (st) (Figure 6C-D). Those fibers in ST ran caudally above the thalamus to project

489 into the amygdala ultimately (Figure 3C-D). In the anterior division of BNST, we observed

490 that the SERT-EGFP fibers were distributed homogeneously in moderately high density (4+)

491 to dense (5+) levels within the anteromedial (am) and anterolateral (al) nuclei. However, the

492 density was mild (2+) in the oval (ov) and fusiform nuclei (fu) (Figure 6A-B). At the posterior

493 division of BNST, we noticed that anteromedial (am), anterolateral (al) and transverse nuclei

494 were very densely (6+) labelled. The rest of the nuclei had dense (5+) labeling except the

495 principal nuclei (pr) which was mildly (2+) labelled except on its ventral part (Figure 6C, D).

\subsection{INNERVATION PATTERN IN THE HIPPOCAMPUS}

497 Hippocampus constitutes of 4 major parts, viz: hippocampal formation (HPF), dentate gyrus 498 (DG), subiculum (SUB) and entorhinal area (ENT). HPF which is also known as cornu 499 ammonis (CA) is further subdivided into various zones, viz; CA1, CA2 and CA3. HPF consists

500 of four different layers; from outward to inward; viz: stratum oriens (SO), pyramidal layer (Py), stratum radiatum (SR), stratum lacunosum molecularae (SLM), whereas, the DG comprises of three layers viz; molecular layer (Mo), granule cell layer (SG) and polymorph layer (Po)

\section{3 (Witter and Amaral, 2004).}

We observed heterogeneously distributed SERT-EGFP fibers among the various layers

505 of hippocampus that gave it a laminar appearance. The SG of dentate gyrus was almost devoid of the innervation, whereas fibers were sparsely $(1+)$ scattered within SP and PO layers (Figure even more dense (6+) in the ventral hippocampus (Figure 7D). The compactly arranged fibers 
510 density (Figure 7B-C). Similarly, the fibers entering into the hippocampus via fimbria (fi) also

511 labelled the CA3 SO in moderately high (4+) density. Therefore, except the CA3 SR and CA3

$512 \mathrm{SO}$, the rest of the areas of these layers and the dorsal part of MO layer were moderately (3+)

513 labelled, whereas the SERT-EGFP fibers were mildly scattered in the ventral part of MO layer

514 (Figure 7B-C). The pattern in the ventral hippocampus was almost congruent with dorsal hippocampus (Figure 7D) except the labelling in SLM was higher than its dorsal counterpart. We traced the fibers of innervation to the hippocampus which were derived through

517 three different sources, viz; dorsal fornix (df), supracallosal bundle (scb) and fimbria (fi). The medially diverted collaterals from the main ascending bundle that traversed through septal nuclei were finally streamed into the scb, df and fi (Figure 7E) (also see the innervation to the septum above). The dorsal fornix was mainly responsible for the innervation of rostral pole of dorsal hippocampus (Figure 7A). The fibers from the fimbria projected into the hippocampus above the $\mathrm{CC}$ and turned around its splenium to terminate finally into the hippocampus (Figure 7C black arrow \& E yellow arrow head). These fibers mainly streamed into the SLM and alveus and were subsequently distributed into the adjacent layers (Figure 7C). We noticed that the collaterals of pathway fibers within the SLM of ventral hippocampus passed externally to get heavily distributed over the rhinal areas (Figure 7D).

\subsection{INNERVATION PATTERN IN THE CORTEX}

529 We traced various routes of innervation of the cerebral cortex which varied depending on the

530 brain levels. At the frontal pole of the brain, the collaterals of pathway fibers clustered within 531 the substantia innominata (SI) passed laterally around the rhinal fissure (RF) to project mainly into cortical layer 1 (Figure 8B-C). Similarly, the fibers clustered within the endopiriform nuclei (EP) also streamed into the layer 1, 5 and $6 \mathrm{~b}$ of lateral cortex (Figure 8C-D). The

534 collateral pathway fibers running in the external capsule (ec) supplemented the innervation of 
535 layer $6 \mathrm{~b}$ and additionally innervated the lateral part of CP (Figure 8C-D, 5B-C arrow). The 536 fibers were very densely (6+) distributed within the claustrum (CLA) (Figure 8C-D). We

537 observed that the medial cortex had separate sources of innervation. It was innervated mainly 538 by the collaterals of pathway fibers streaming through supracallosal bundle (scb) (Figure 7E,

539 8D \& E), indusium griseum (ig) (Figure 8C \& H, arrow), and medial cortical bundle (Figure

540 8C). We noticed that the outermost cortical layer was the most labelled (5+) layer having transversely oriented fibers to the horizontal axis. We observed difference in the innervation pattern among the various cortical areas and gradual decrease in the labelling density as traced rostro-caudally.

\section{$544 \quad$ 3.9.1 Prefrontal Cortex}

545 The prefrontal cortex (PFC) of the mouse consists of three major parts, the medial (mPFC), orbital (ORB) and agranular insular cortices (AI). mPFC is further subdivided into prelimbic

547 (PL), infralimbic (IL) and anterior cingulate area (ACA) (Allen Institute for Brain Science, 2004). We observed that these different areas exhibited heterogeneous innervation density and pattern along the rostro-caudal axis. At the rostral pole, the PL area had patch like fibers

550 clustered within the upper layers in moderately high (4+) density (Figure 8A). However, the caudal extent of PL area (Figure 8C) and the whole rostro-caudal extent of ILA and ACA (Figure 8A-D) were moderately (3+) labelled except at layer 1 (Figure 8B-D). The fibers that entered into the mPFC via EP nuclei (Figure 8B) and medial cortical bundle (Figure 8C) ran vertically through its deeper layers. Similarly, the orbitofrontal cortex (ORB) exhibited an alternating laminar pattern towards its rostral extent (Figure 8A) which was less evident caudally especially on its medial (m) part (Figure 8B). Innervation of AI also appeared in a laminar pattern because of the readily identifiable layer 1 and 5 which received higher innervation compared to the intervening layer (Figure 8A-C). Traced more caudally, the 
deepest layer (layer 6b) in AI cortex was also densely (5+) labelled because of fibers entering

560 into the lateral cortex through layer 1, 5 and $6 \mathrm{~b}$ (Figure 8D).

\subsubsection{Retrosplenial cortex}

562 The retrosplenial cortex (RSP) contained a distinct vertical band of fibers running in parallel to layer 1 at its rostral pole (Figure $8 \mathrm{E} \& \mathrm{~F}$ ). The fibers were moderately $(3+)$ distributed (Figure 8E) which progressively decreased in density caudally (Figure 8F-G). The fibers clustered within the supracallosal bundle ( $\mathrm{scb}$ ) was the source of innervation to the RSP cortex (Figure $8 \mathrm{E}$, arrow).

\subsubsection{Motor cortex}

Motor cortex also exhibited the laminar innervation pattern. Similar to the other cortical areas,

569 layer 1 was distinct because of its dense (5+) innervation. Towards the rostral pole, layer 5 of the primary motor cortex (MOp) was densely (5+) labelled (Figure $8 \mathrm{~A})$, which gradually decreased in density (4+) as traced caudally (Figure $8 \mathrm{~B}-\mathrm{E}$ ). The rest of the layers were moderately $(3+)$ labelled, thus giving it a laminar appearance. The fibers in layer $2 / 3$ were oriented vertically to the horizontal axis, whereas randomly distributed elsewhere (Figure 8DE). The MOp and secondary motor (MOs) area exhibited slight differences in the innervation density only at its rostral pole (Figure 8A), whereas the patterns were almost similar elsewhere (Figure 8B-E).

\subsubsection{Somatosensory cortex}

578 Rostral half of somatosensory (SS) cortex appeared distinct from the adjacent motor (MO) 579 cortex because of its densely (5+) labelled layer 5 and mildly (2+) labelled layer $2 / 3$ and 4 580 (Figure 8B-D). However, the innervation density of layer 5 gradually decreased to moderately

581 high (4+) level towards its caudal extent (Figure 8E). Thus, SS cortex appeared similar to the

582 adjacent MO cortex at the caudal level (Figure 8E). We observed that the collaterals of pathway 583 fibers passing within the external capsule (ec) and claustrum (CLA) densely (5+) arborized in 
the barrel field (bf) area (Figure 8D). The higher labelling of layer 1, 5 and $6 \mathrm{~b}$ compared to the intervening layers made the innervation pattern alternatingly laminar in SS cortex (Figure 8BE). Basically, we found no difference in innervation pattern between the primary and secondary

587 SS cortex. The overall innervation density in the SS cortex decreased rostro-caudally.

\subsubsection{Auditory and visual cortexes}

589 The auditory and visual cortices which are located towards the caudal pole of the mouse brain received relatively less serotonergic innervation compared to the rostrally located cortical regions like the motor and somatosensory cortices (Figure 8F-G \& 7D). The outer most layer 1 was thin but densely (5+) innervated. The underlying layer $2 / 3$ and 4 were sparsely $(1+)$ innervated (Figure 8F-G \& 7D), however, the innervation density in rest of the layers decreased

594 from moderate $(3+)$ labeling in the rostral half (Figure 8F-G) to milder $(2+)$ density towards the caudal end (Figure 7D). Thus, the overall innervation density in the auditory and visual cortex also progressively decreased rostro-caudally (Figure 8F-G \& 7D).

\subsubsection{Rhinal Area}

598 The rhinal cortex was the most labelled cortical area. At its rostral extent, all the layers except

599 the layer 2/3 were densely (5+) innervated (Figure 8F). However, towards its caudal pole, layer $2 / 3$ and the deepest layer were mildly $(2+)$ innervated while the others received very dense $(6+)$ innervation (Figure 7D). We observed that the collaterals of pathway fibers running across the stratum lacunosum molecularae ( $\operatorname{sim})$ of the ventral hippocampus moved outside of it to

603 heavily innervate the laterally located rhinal cortex (Figure 7D).

\section{$604 \quad$ 3.9.7 Piriform cortex}

605 In this three-layered structure, the rostral extent of piriform cortex (PIR) was moderately (3+)

606 labelled without laminar appearance (Figure 8D). However, a laminar pattern was observed in

607 the caudal PIR where the outer layer was densely (5+) labelled and appeared distinct from 608 underlying layer 2 and 3 which were moderately (3+) labelled (Figure 3C). 


\subsection{INNERVATION PATTERN IN THE OLFACTORY TUBERCLE and} OLFACTORY BULB

611 We observed densely (5+) distributed thick and tortuous fibers clustered around the island of

612 Calleja (isl) within the rostral extent of olfactory tubercle (OT) (Figure 6A-B), while the isl

613 being almost devoid of the innervation. However, the caudal OT was innervated with

614 homogenously distributed fine fibers in slightly less density compared to its rostral counterparts

615 (Figure 6C-D). The OT and SI served as the route for the ascending forebrain bundle to traverse

616 rostrally towards the olfactory bulb (Figure 6A).

617 Main olfactory bulb (MOB) was the terminal projection site of the ascending forebrain

618 bundle arising from the midbrain raphe nuclei (Figure 9A). Fibers in MOB were arranged in a

619 laminar pattern. We noticed that the outer most glomerular (GL) layer was densely (5+) innervated with SERT-EGFP fibers. Mitral (MI), internal plexiform (iPL) and granule (GR) moderately high $(4+)$ density. The outer plexiform layer (OPL) and olfactory ventricles (OV) were sparsely $(1+)$ labelled. We noticed that glomerular fibers were thick in diameter, intensely labelled and contained large varicosities, while thinner fibers predominated in the infraglomerular layers. The olfactory nerve layer (onl) was completely devoid of the serotonergic projections (Figure 9A-B).

The accessory olfactory bulb (AOB) was devoid of the innervation, except the few 628 sparsely $(1+)$ scattered fibers within the granular (gr) layer (Figure 9B).

\subsection{INNERVATION PATTERN IN THE CEREBELLUM}

630 Cerebellum was the least innervated major brain component (Figure 10). Few fibers (1+) were 631 distributed within the Purkinje cell layer (pr) and granular layer (gr). Molecular layer (mo) and 632 white matter (wm) appeared unlabeled. Surprisingly, we observed that deep cerebellar nuclei 633 were moderately $(3+)$ labelled because of the direct projection from the raphe nuclei. 
634 The results for each brain region and their nuclei are tabulated in the table 2.

635

636

637

638

639

640

641

642

643

644

645

646

647

648

650

651

652

653

654

655

656

657

658

\section{DISCUSSION}

The serotonergic system is one of the diffusively organized brain neuronal systems. Although the cellular localization and projections map was first described long ago with the advent of histofluorescence technique (Dahlström and Fuxe, 1964), the improved topographical description was possible only after the development of antibodies against the enzyme tryptophan hydroxylase (Pickel et al., 1977) or the putative neurotransmitter 5-HT itself (Steinbusch, 1981). However, these techniques were accompanied with certain limitations. In order to overcome the limitations of earlier studies, we employed the genetically engineered transgenic mice expressing GFP in the 5-HT transporter. Using this mouse model, we attempted to provide more comprehensive map of the terminal field of serotonergic neurons than is currently available. In the following section, we highlight some of our results, compare them with those of previous studies and discuss the possible functional implications.

The serotonergic neurons projecting to the forebrain originated from the rostral group of raphe nuclei in the midbrain and virtually innervated all the brain regions with striking density in the hypothalamus, septal nuclei, thalamus, amygdala, olfactory bulb followed by basal nuclei and cortex. Except the white matter structures, there was hardly no brain region that did not receive 5-HT innervation. This was consistent with the previous findings (Steinbusch, 1981). We observed two different types of innervation fibers; fibers with large and spherical varicosities and fine fibers with small and granule shaped varicosities. Previous studies have shown that the difference in fiber morphology possesses functional significance. For instance, the fine 5-HT axon terminals are supposed to be extremely vulnerable to psychotropic drugs like amphetamine (O’Hearn et al., 1988). Similarly, change in the ratio of these fiber types has been observed in epilepsy where fibers with small-sized varicosities were decreased in the dentate gyrus of hippocampus, infralimbic cortex and medial septum while 
that of the fibers with larger-sized varicosities were increased (Maia et al., 2019). Moreover,

660 changes in the morphology of serotonergic fibers associated with aging have been reported

661 (Nishimura et al., 1998). Thus, analysis of 5-HT structural system could help understand the

662 pathophysiology of mental disorders and lead to drug discovery. The 5-HT projection

663 demonstrated an extensive and very specific innervation pattern in the brain areas as discussed

664 below.

\section{$665 \quad 4.1$ Thalamus}

666 SERT-EGFP fibers were mainly concentrated in midline thalamus (paraventricular, paratenial,

667 rhomboid and reuniens nuclei), rostral part of intralaminar thalamus (central medial and central

668 lateral nuclei), some part of anterior thalamus (anteroventral, rostral part of intermediodorsal

669 nuclei) and in the other nuclei like lateral dorsal nucleus (LD), lateral posterior nucleus (LP)

670 and lateral geniculate (LG) complex etc.

671 Most of the previous findings of thalamic mapping using peroxidase-antiperoxidase

672 (PAP) technique were consistent to ours (Cropper et al., 1984). However, they reported light

673 labeling in several nuclei like mediodorsal, centromedian, and subthalamic nuclei where we

674 found fibers in comparatively higher densities. Conversely, we found very light labelling in caudal part of reticular nuclei, ventral medial nuclei and posterior nuclei which they reported to be moderately labelled. Similar to us, other studies have also reported that principal thalamic

677 nuclei lack the serotonergic input (Vertes et al., 1999). We observed very distinct distribution

678 of fibers across the rostro-caudal axis of the reticular nuclei that progressively decreased in 679 their density towards their caudal pole. This contrasts with the findings of one of the 680 immunohistochemical studies which reported to have little difference (Rodríguez et al., 2011).

681 We noticed some species differences as well. Similar to mice, the non-specific nuclei in primate 682 (squirrel monkey) received the heaviest innervation. However, the lightly labelled reticular 
683

684

685

686

687

688

689

690

691

692

693

694

695

696

697

698

699

700

701

702

703

704

705

706

707

nuclei in mice were heavily labelled in the monkey, whereas, the richly innervated nuclei in mice such as AV, LD, and LGd were less innervated in the monkey (Lavoie and Parent, 1991). Studies have shown that the midline thalamic nuclei in connection with limbic subcortical and cortical sites (Su and Bentivoglio, 1990) exert an arousing effect on the limbic forebrain (Vertes, 2006). The high density of 5-HT innervation in these nuclei suggests that they might modulate the emotional and cognitive functions. Similarly, the proposal of anterior thalamic nuclei as an extended component hippocampal-dependent memory network, (Aggleton and Brown, 1999) suggests that high serotonergic innervation in these nuclei might exert some effect on episodic memory. Projections of large numbers of SERT-EGFP fibers to the lateral dorsal nuclei (LD) and lateral geniculate nuclei suggest that it might modulate visually guided spatial navigation and learning (Mizumori et al., 1994) or may sharpen the visuospatial processing activity (Groenewegen and Witter, 2004). Similarly, studies have reported that the downregulation of serotonergic system in the lateral habenula is linked with the depressive symptoms in patients with Parkinson's disease (Sourani et al., 2012). However, the precise role served by 5 -HT in various thalamic nuclei and their function remains to be fully determined.

\subsection{Hypothalamus}

We observed that the hypothalamic nuclei received strong SERT-EGFP fiber input with exceptions in some nuclei. The ascending forebrain bundle traversed rostrally through the lateral hypothalamic area (LHA) and lateral preoptic (LPO) nuclei which made them appear over crowded. There are some older reports showing 5-HT projections in hypothalamus. However, some of them had employed older autoradiographic technique and did not cover all the nuclei (Beaudet and Descarries, 1979), while another had used antibody against 5-HT but without the monoamine oxidase inhibitor treatment (Steinbusch, 1981). Although the patterns reported in many of the nuclei were similar to us, we observed higher labelling density in many 
708

709

710

711

712

713

714

715

716

717

718

719

720

721

722

723

724

725

726

727

728

729

730

731

of the nuclei than their reports because of the superiority of the technique used. None of these previous studies have reported the changes in the labelling pattern in the different hypothalamic nuclei across the rostro-caudal axis.

Some earlier studies have reported the presence of serotonergic cell bodies in the dorsomedial hypothalamic nucleus of rats (Fuxe et al., 1968) (Beaudet and Descarries, 1979) but we did not detect any such cell bodies in hypothalamus. We think that this might be a technical limitation in autoradiographic technique where some non-specific neurons might have absorbed infused 5-HT. A study employing antibody against 5-HT in guinea pig brain also did not detect such cell bodies in hypothalamus (Warembourg and Poulain, 1985).

The ventromedial part of the SCN was one of the highest labelled nuclei in the whole brain. The finding corroborates with results from other rodent models such as hamster (Legutko and Gannon, 2001) and rat (Steinbusch, 1981), and is also consistent with humans (Borgers et al., 2014). According to the earlier tracing studies, SCN receives very dense projections from the median raphe (MR), but not from the DR (Meyer-Bernstein and Morin, 1996) (Muzerelle et al., 2016). Therefore, it can be hypothesized that depletion of 5-HT in the MR or the destruction of 5-HT fibers restricted to the SCN could affect the circadian rhythm. Some previous reports have shown that 5-HT activity on the SCN inhibits the effects of light on the circadian system (Meyer-Bernstein and Morin, 1996) (Bradbury et al., 1997). Moreover, it is reported that the release of 5-HT at the SCN follows the daily rhythm and the behavioral state can strongly influence the serotonergic activity in the circadian clock (Dudley et al., 1998). This strong innervation of 5-HT fibers to the SCN suggests the reciprocal connections of the 5-HT and circadian systems and they may have importance for neurodevelopmental and psychiatric disorders such as ASD and mood disorders, respectively (Ciarleglio et al., 2011) (Takumi et al., 2019). 
ventromedial hypothalamic and paraventricular nuclei exhibited both rostro-caudal and intranuclear innervation gradients. The lateral hypothalamic area was too crowded because of the ascending forebrain bundle traversing through it. However, how these wide variations in 5HT-innervation of these nuclei affect the hunger mechanism is yet to be fully understood. Some intake (Lam et al., 2010).

\subsection{Amygdala}

742 We observed that most of the amygdalar nuclei received SERT-EGFP fibers in high density except the few nuclei such as lateral amygdala (LA), central amygdala (CeA) and medial amygdala (MeA) were comparatively less innervated. Some of the previous studies have reported serotonergic innervation patterns in the amygdaloid complex using various techniques such as autoradiography (Parent et al., 1981), antibodies against 5-HT (Steinbusch, 1981) or 5HT transporter (Sur et al., 1996), in situ hybridization (Bonn et al., 2013) and electron microscopy (Muller et al., 2007). However, most of them had either studied only the selected amygdaloid nuclei or have reported lighter density compared to us which might be due to the technical limitations. However, the common finding was that 5-HT fibers distribute densely to the BLA and BMA but less heavily to the LA and CeA nuclei. This was also consistent with the findings reported in a recent review (Asan et al., 2013). In addition, a recent study also showed that BLA is the most labelled nuclei among the different limbic structures which are in the sequence of BLA $>$ NAc $>$ BNST $>$ HIP $>$ CeA $>$ mPFC (Belmer et al., 2017). An anterograde tracing study has reported that amygdalar nuclei receive serotonergic efferents mainly from the DR and very minor projections from the MR (Muzerelle et al., 2016). 
fibers. On contrary to the rodent pattern, the fiber densities in non-human primate were in the order of central nucleus $>$ basolateral complex $>$ medial nucleus (O’Rourke and Fudge, 2006) (Zeng et al., 2006), while in the human amygdala sequence followed: cortical and anterior amygdaloid nuclei $>$ basolateral and central nuclei (Storvik et al., 2007). Differential expression of the SERT has been linked to species variation in sensitivity to social cues, vigilance to social threats, risk avoidance, responsiveness to changes in reward contexts and mood (Vallender et al., 2009). modify the information received from LA to CeA nuclei (Amano et al., 2011). Coupling this fact with the presence of very dense 5-HT innervation suggests that it could be the primary amygdalar target for 5-HT neuromodulation of fear and anxiety. This is supplemented by reduces anxiety and fear (Johnson et al., 2015). Similarly, action of oxytocin on the MeA nuclei in facilitating the social recognition (Ferguson et al., 2001) could be coupled with the presence of heavy 5-HT innervation in MEAad as the common target for both in modulating the social recognition. Additionally, the decrease in density of 5-HT axons in the CeA and BLA and in the CA3 of the hippocampus due to postnatal social isolation has been linked to depression (Kuramochi and Nakamura, 2009).

\subsection{Basal Ganglia}

777 We observed fibers in higher density in globus pallidus (GP) compared to caudo-putamen (CP) which contrast with the findings of earlier immunohistochemical studies where no difference in the distribution density was reported (Steinbusch, 1981). Within the CP, fiber densities were 
782

783

784

785

786

787

788

789

790

791

792

793

794

795

796

797

798

799

800

801

802

803

804

805

806

1977) we observed that the innervation density in the striatum increases rostro-caudally. The distribution density of SERT-EGFP fibers demonstrated in our study was consistent with the amount of 5-HT detected in different parts of striatum by liquid chromatography (Beal and Martin, 1985). Similarly, we noticed higher SERT-EGFP fiber density in the external segment (GPe) than the internal segment (GPi) of GP. This pattern was consistent even in primates (Eid et al., 2013). Studies have reported that the innervation pattern in striatum is shared in both rodent and non-human primates (Mori et al., 1985). A recent tracing study showed that striatum and GP receive projection mainly from the supralemiscal (B9) group and minorly from the DR and MR (Muzerelle et al., 2016).

The high serotonergic innervation in the GP suggests that it might be the pivotal site for the 5-HT involvement in the basal ganglia functions or pathophysiology, although it is yet to be confirmed. However, a study has shown that 5-HT influences the reward seeking circuitry involving GPi and lateral habenula (Hong and Hikosaka, 2008). Similarly, the result from one of the studies showing the preferential loss of striatal SERT fibers in Parkinson disease (PD) (Kish et al., 2007) indicates the involvement of 5-HT along with dopamine in the PD pathophysiology. A recent study showed that 5-HT affects the synaptic signaling at thalamostriatal inputs (Cavaccini et al., 2018) which suggests that striatal-dependent functions may be subjected to serotonergic modulation.

\subsection{Cortex}

We found densely concentrated SERT-EGFP fibers in the alternating layers of cortical areas such as mPFC, insular cortex, somatosensory cortex, piriform and rhinal cortices. This was in contrary to the previous immunohistochemical report where they mentioned the uniform distribution of fibers across all the cortical layers (Lidov et al., 1980). Similar to us, one of the immunohistochemical studies reported that the elaborated radial plexus innervates the upper layers and the tangential fibers course through the deeper layers of prelimbic cortex (Miner et 
al., 2000). We noticed that prelimbic cortex that projects to the limbic areas received higher

808 projection fibers compared to non-limbic regions (i.e. ACC). This could possibly explain the

809 underlying role of 5-HT on the emotional, behavioral and cognitive functions. Any alteration

810 could possibly subserve the pathophysiology of many mental disorders. For instance,

811 reductions in the length of SERT fibers in the orbitofrontal cortex have been found in the

812 postmortem brain of the patient with major depressive disorder (Rajkowska et al.,

813 2017). Similarly, reduced SERT-ir fiber density in the medial frontal cortex, midbrain, and

814 temporal lobe areas have been reported in the autistic brain (Makkonen et al., 2008). A study

815 that employed radiographic techniques reported the prelimbic and rostral agranular insula as

816 the most densely labeled cortical areas sites (Audet et al., 1989). However, in our case we

817 noticed that some other areas like somatosensory (layer 5) and rhinal cortices were equally or

818 even more densely innervated compared to PLA and AI. Anterograde tracing study has shown

819 that both DR and MR projects to prefrontal cortex with DR being the primary source

820 (Muzerelle et al., 2016).

Next, we noticed species difference in the cortical layer labelling density. In our rodent model, a lightly labelled layer $2 / 3$ intervene between moderately labelled superficial and deeper layers of the visual cortex. In the adult cat visual cortex, dense fibers were distributed within the layers I and III (Gu et al., 1990). In monkey, layer IV was the highest labelled layer visual cortical layer, whereas other layers received either less dense or very sparse labelling (Morrison et al., 1982).

Similarly, we observed dense projection within the barrel field area of the somatosensory cortex suggesting its significant role in modulating sensory information. Developmental studies have already demonstrated that 5-HT plays a significant role in the 830 development of barrel formation in cortex (Persico et al., 2000). Not limited to the barrel field, 831 compelling evidence suggests that 5-HT is necessary for the maturation, dendritic arborization, 
migration, differentiation of many different kinds of neurons and interneurons which are

833 essential for the refined organization of the cerebral cortex (Vitalis et al., 2007).

\section{5. Conclusion}

835 In conclusion, we mapped the distribution pattern of the serotonergic neurons across the whole

836 brain region using SERT-EGFP model mice. The use of transgenic animal helped to elucidate

837 the topography of serotonergic system in much greater detail. We identified higher density of

838 projections than previously reported and observed that the densities and pattern changes along

839 the rostro-caudal brain axis. Although serotonergic fibers were ubiquitously distributed in the

840 brain, there was a defined topographic organization of these projections with strikingly high

841 projections in some specific targets. With a couple of exceptions, many of the nuclei with high serotonergic projections were anatomically linked to forebrain limbic structures, suggesting that 5-HT has modulatory effects on emotional and cognitive behaviors. A detailed analysis of

844 the topographical distribution of these neuronal populations will provide an anatomical basis to postulate the physiological role of 5-HT in different behavior and in the understanding of

846 possible alteration in numerous mental and psychiatric disorders.

\section{$848 \quad$ Acknowledgement}

849 The authors express sincere thanks to Tsuyoshi Toya, Jun Nomura and Takeshi Kaizuka for 850 their useful help and discussions and all the technical staff of the Takumi Lab for their technical 851 assistance.

\section{Authors contribution}

853 All authors together conceived and designed the experiments. JRA drafted the manuscript, 854 collected the data and carried out the analysis with advice from KT and TT. All authors were 855 involved in the discussion and editing of the final manuscript. TT supervised the project.

856 Competing Interests: The authors declare that they have no competing interests. 


\section{References}

858 Abe K, Shimada R, Okada Y, Kibayashi K. 2016. Traumatic brain injury decreases serotonin transporter expression in the rat cerebrum. Neurol Res 38:358-363.

860 Aggleton JP, Brown MW. 1999. Episodic memory, amnesia, and the hippocampal-anterior thalamic axis. Behav Brain Sci 22:425-44; discussion 444-89.

862 Allen Institute for Brain Science. 2004. Allen Mouse Brain Atlas. Available from: http://atlas.brain-map.org/

Amano T, Duvarci S, Popa D, Pare D. 2011. The Fear Circuit Revisited: Contributions of the Basal Amygdala Nuclei to Conditioned Fear. J Neurosci 31:15481-15489.

Asan E, Steinke M, Lesch KP. 2013. Serotonergic innervation of the amygdala: Targets, receptors, and implications for stress and anxiety. Histochem Cell Biol 139:785-813.

Audet MA, Descarries L, Doucet G. 1989. Quantified regional and laminar distribution of the serotonin innervation in the anterior half of adult rat cerebral cortex. J Chem Neuroanat 2:29-44.

Azmitia E, Gannon P. 1983. The ultrastructural localization of serotonin immunoreactivity in myelinated and unmyelinated axons within the medial forebrain bundle of rat and monkey. J Neurosci 3:2083-90.

Beal MF, Martin JB. 1985. Topographical dopamine and serotonin distribution and turnover in rat striatum. Brain Res 358:10-15.

876 Beaudet A, Descarries L. 1979. Radioautographic characterization of a serotonin-accumulating nerve cell group in adult rat hypothalamus. Brain Res 160:231-243.

878 Belmer A, Klenowski PM, Patkar OL, Bartlett SE. 2017. Mapping the connectivity of serotonin transporter immunoreactive axons to excitatory and inhibitory neurochemical synapses in the mouse limbic brain. Brain Struct Funct 222:1297-1314.

881 Berger M, Gray JA, Roth BL. 2009. The Expanded Biology of Serotonin. Annu Rev Med 
883 Bonn M, Schmitt A, Lesch K-P, Van Bockstaele EJ, Asan E. 2013. Serotonergic innervation 884 and serotonin receptor expression of NPY-producing neurons in the rat lateral and 885 basolateral amygdaloid nuclei. Brain Struct Funct 218:421-435.

886 Borgers AJ, Koopman KE, Bisschop PH, Serlie MJ, Swaab DF, Fliers E, la Fleur SE, 887 Alkemade A. 2014. Decreased serotonin transporter immunoreactivity in the human hypothalamic infundibular nucleus of overweight subjects. Front Neurosci 8:106.

Bradbury MJ, Dement WC, Edgar DM. 1997. Serotonin-containing fibers in the suprachiasmatic hypothalamus attenuate light-induced phase delays in mice. Brain Res $768: 125-34$.

Cavaccini A, Gritti M, Giorgi A, Locarno A, Heck N, Migliarini S, Bertero A, Mereu M, Margiani G, Trusel M, Catelani T, Marotta R, De Luca MA, Caboche J, Gozzi A, Plasticity at Striatal Circuits. Neuron 98:801-816.e7.

Ciarleglio CM, Resuehr HES, McMahon DG. 2011. Interactions of the serotonin and circadian systems: Nature and nurture in rhythms and blues. Neuroscience 197:8-16.

Cropper EC, Eisenman JS, Azmitia EC. 1984. An immunocytochemical study of the serotonergic innervation of the thalamus of the rat. J Comp Neurol 224:38-50. , 091). Los Angeles.

Dahlström A, Fuxe K. 1964. Localization of monoamines in the lower brain stem. Experientia 20:398-9.

Dudley TE, DiNardo LA, Glass JD. 1998. Endogenous regulation of serotonin release in the hamster suprachiasmatic nucleus. J Neurosci 18:5045-52.

Eid L, Champigny M-F, Parent A, Parent M. 2013. Quantitative and ultrastructural study of 

1668.

Ferguson JN, Aldag JM, Insel TR, Young LJ. 2001. Oxytocin in the medial amygdala is essential for social recognition in the mouse. J Neurosci 21:8278-85.

Fuxe K. 1965. Evidence for the existence of monoamine neurons in the central nervous system. Iv. Distribution of monoamine nerve terminals in the central nervous system. Acta Physiol Scand Suppl:SUPPL 247:37+.

914 Fuxe K, Hökfelt T, Ungerstedt U. 1968. Localization of indolealkylamines in CNS. Adv Pharmacol 6:235-51.

916 Gong S, Zheng C, Doughty ML, Losos K, Didkovsky N, Schambra UB, Nowak NJ, Joyner A,

917 Leblanc G, Hatten ME, Heintz N. 2003. A gene expression atlas of the central nervous system based on bacterial artificial chromosomes. Nature 425:917-925.

919 Groenewegen HJ, Witter MP. 2004. Thalamus. Rat Nerv Syst:407-453.

Gu Q, Patel B, Singer W. 1990. The laminar distribution and postnatal development of serotonin-immunoreactive axons in the cat primary visual cortex. Exp brain Res 81:25766.

Harrington B. 2005. Draw Freely | Inkscape. Available from: https://inkscape.org/ Habenula. Neuron 60:720-729.

Hrovatin K, Kunej T, Dolžan V. 2019. Genetic variability of serotonin pathway associated with schizophrenia onset, progression, and treatment. Am J Med Genet Part B Neuropsychiatr Genet. 
932 Johnson PL, Molosh A, Fitz SD, Arendt D, Deehan GA, Federici LM, Bernabe C, Engleman

933 EA, Rodd ZA, Lowry CA, Shekhar A. 2015. Pharmacological depletion of serotonin in

934 the basolateral amygdala complex reduces anxiety and disrupts fear conditioning.

935 Pharmacol Biochem Behav 138:174-179.

936 Kish SJ, Tong J, Hornykiewicz O, Rajput A, Chang L-J, Guttman M, Furukawa Y. 2007.

937 Preferential loss of serotonin markers in caudate versus putamen in Parkinson's disease.

$938 \quad$ Brain 131:120-31.

939 Kuramochi M, Nakamura S. 2009. Effects of postnatal isolation rearing and antidepressant

940 treatment on the density of serotonergic and noradrenergic axons and depressive behavior

941 in rats. Neuroscience 163:448-455.

942 Lam DD, Garfield AS, Marston OJ, Shaw J, Heisler LK. 2010. Brain serotonin system in the coordination of food intake and body weight. Pharmacol Biochem Behav 97:84-91.

944 Lavoie B, Parent A. 1991. Serotoninergic innervation of the thalamus in the primate: An immunohistochemical study. J Comp Neurol 312:1-18.

946 Legutko R, Gannon RL. 2001. Serotonin transporter localization in the hamster 947 suprachiasmatic nucleus. Brain Res 893:77-83.

948 Lidov HGW, Grzanna R, Molliver ME. 1980. The serotonin innervation of the cerebral cortex in the rat-an immunohistochemical analysis. Neuroscience 5:207-227.

Lin SH, Lee LT, Yang YK. 2014. Serotonin and mental disorders: A concise review on molecular neuroimaging evidence. Clin Psychopharmacol Neurosci 12:196-202.

952 Maciag D, Simpson KL, Coppinger D, Lu Y, Wang Y, Lin RCS, Paul IA. 2006. Neonatal antidepressant exposure has lasting effects on behavior and serotonin circuitry. Neuropsychopharmacology 31:47-57.

Maia GH, Soares JI, Almeida SG, Leite JM, Baptista HX, Lukoyanova AN, Brazete CS, Lukoyanov N V. 2019. Altered serotonin innervation in the rat epileptic brain. Brain Res 

Bull 152:95-106.

Makkonen I, Riikonen R, Kokki H, Airaksinen MM, Kuikka JT. 2008. Serotonin and dopamine transporter binding in children with autism determined by SPECT. Dev Med Child Neurol 50:593-597.

McDonald AJ. 1998. Cortical pathways to the mammalian amygdala. Prog Neurobiol 55:257-

962 332.

Meyer-Bernstein EL, Morin LP. 1996. Differential serotonergic innervation of the suprachiasmatic nucleus and the intergeniculate leaflet and its role in circadian rhythm modulation. J Neurosci 16:2097-111.

966

Miner LH, Schroeter S, Blakely RD, Sesack SR. 2000. Ultrastructural localization of the serotonin transporter in superficial and deep layers of the rat prelimbic prefrontal cortex and its spatial relationship to dopamine terminals. J Comp Neurol 427:220-34.

Mizumori SJY, Miya DY, Ward KE. 1994. Reversible inactivation of the lateral dorsal thalamus disrupts hippocampal place representation and impairs spatial learning. Brain Res 644:168-174.

Mori S, Ueda S, Yamada H, Takino T, Sano Y. 1985. Immunohistochemical demonstration of serotonin nerve fibers in the corpus striatum of the rat, cat and monkey. Anat Embryol (Berl) 173:1-5.

Morrison JH, Foote SL, Molliver ME, Bloom FE, Lidov HG. 1982. Noradrenergic and serotonergic fibers innervate complementary layers in monkey primary visual cortex: an

Muller JF, Mascagni F, McDonald AJ. 2007. Serotonin-immunoreactive axon terminals innervate pyramidal cells and interneurons in the rat basolateral amygdala. J Comp Neurol 505:314-335. 

to the forebrain and brainstem. Brain Struct Funct 221:535-561.

984

985

986

987

988

989

990

991

992

993

994

995

996

997

998

999

1000

1001

1002

1003

1004

1005

1006

Nielsen K, Brask D, Knudsen GM, Aznar S. 2006. Immunodetection of the serotonin transporter protein is a more valid marker for serotonergic fibers than serotonin. Synapse 59:270-276.

Nishimura A, Ueda S, Takeuchi Y, Matsushita H, Sawada T, Kawata M. 1998. Vulnerability to aging in the rat serotonergic system. Acta Neuropathol 96:581-595.

O’Hearn E, Battaglia G, De Souza EB, Kuhar MJ, Molliver ME. 1988. Methylenedioxyamphetamine (MDA) and methylenedioxymethamphetamine (MDMA) cause selective ablation of serotonergic axon terminals in forebrain: immunocytochemical evidence for neurotoxicity. J Neurosci 8:2788-803.

O’Rourke H, Fudge JL. 2006. Distribution of Serotonin Transporter Labeled Fibers in Amygdaloid Subregions: Implications for Mood Disorders. Biol Psychiatry 60:479-490.

Okaty BW, Commons KG, Dymecki SM. 2019. Embracing diversity in the 5-HT neuronal system. Nat Rev Neurosci 20:397-424.

Parent A, Descarries L, Beaudet A. 1981. Organization of ascending serotonin systems in the adult rat brain. A radioautographic study after intraventricular administration of [3h]5hydroxytryptamine. Neuroscience 6:115-138.

Persico AM, Altamura C, Calia E, Puglisi-Allegra S, Ventura R, Lucchese F, Keller F. 2000. Serotonin Depletion and Barrel Cortex Development: Impact of Growth Impairment vs. Serotonin Effects on Thalamocortical Endings. Cereb Cortex 10:181-191.

Pickel VM, Joh TH, Reis DJ. 1977. A serotonergic innervation of noradrenergic neurons in nucleus locus coeruleus: demonstration by immunocytochemical localization of the transmitter specific enzymes tyrosine and tryptophan hydroxylase. Brain Res 131:197214. 
Pollak Dorocic I, Fürth D, Xuan Y, Johansson Y, Pozzi L, Silberberg G, Carlén M, Meletis K. 2014. A Whole-Brain Atlas of Inputs to Serotonergic Neurons of the Dorsal and Median Raphe Nuclei. Neuron 83:663-678.

Rajkowska G, Mahajan G, Legutko B, Challagundla L, Griswold M, Albert PR, Daigle M, Miguel-Hidalgo JJ, Austin MC, Blakely RD, Steffens DC, Stockmeier CA. 2017. Length of axons expressing the serotonin transporter in orbitofrontal cortex is lower with age in depression. Neuroscience 359:30-39.

Rasband W. 1997. ImageJ. U S Natl Institutes Heal Bethesda, Maryland, USA, [Internet]. Available from: https://imagej.nih.gov/ij/

Reinebrant H, Wixey J, Buller K. 2020. Hypoxia-ischemia in the immature rodent brain impairs serotonergic neuronal function in certain dorsal raphé nuclei. Neural Regen Res 15:457463.

Ren J, Friedmann D, Xiong J, Liu CD, Ferguson BR, Weerakkody T, DeLoach KE, Ran C,

Ren J, Isakova A, Friedmann D, Zeng J, Grutzner SM, Pun A, Zhao GQ, Kolluru SS, Wang R, transcriptomes and whole-brain projections of serotonin neurons in the mouse dorsal and median raphe nuclei. Elife 8 .

Risold PY. 2004. The Septal Region. In: The Rat Nervous System. Elsevier Inc. p 605-632.

Rodríguez JJ, Noristani HN, Hoover WB, Linley SB, Vertes RP. 2011. Serotonergic projections and serotonin receptor expression in the reticular nucleus of the thalamus in the rat. Synapse 65:919-928.

1031 Schmidt EF, Kus L, Gong S, Heintz N. 2013. BAC Transgenic Mice and the GENSAT 
1032 Database of Engineered Mouse Strains.

1033 Sourani D, Eitan R, Gordon N, Goelman G. 2012. The habenula couples the dopaminergic and 1034 the serotonergic systems: application to depression in Parkinson's disease. Eur J Neurosci $1035 \quad 36: 2822-2829$.

1036 Steinbusch HW. 1981. Distribution of serotonin-immunoreactivity in the central nervous system of the rat-cell bodies and terminals. Neuroscience 6:557-618.

Storvik M, Tiihonen J, Haukijärvi T, Tupala E. 2007. Amygdala serotonin transporters in alcoholics measured by whole hemisphere autoradiography. Synapse 61:629-636.

$1040 \mathrm{Su}$ H-S, Bentivoglio M. 1990. Thalamic midline cell populations projecting to the nucleus accumbens, amygdala, and hippocampus in the rat. J Comp Neurol 297:582-593.

Sur C, Betz H, Schloss P. 1996. Immunocytochemical detection of the serotonin transporter in rat brain. Neuroscience 73:217-31.

1044 Takumi T, Tamada K, Hatanaka F, Nakai N, Bolton PF. 2019. Behavioral neuroscience of autism. Neurosci Biobehav Rev 110:60-76.

1046 Tamada K, Takumi T. 2015. Serotonin disturbance in mouse models of autism spectrum disorders. In: Organism Models of Autism Spectrum Disorders. Springer New York. p $239-262$.

Ternaux JP, Héry F, Bourgoin S, Adrien J, Glowinski J, Hamon M. 1977. The topographical

Vallender EJ, Lynch L, Novak MA, Miller GM. 2009. Polymorphisms in the 3' UTR of the serotonin transporter are associated with cognitive flexibility in rhesus macaques. Am $\mathbf{J}$ Med Genet Part B Neuropsychiatr Genet 150:467-475. thalamus in emotional and cognitive processing in the rat. Neuroscience 142:1-20. 
Vertes RP, Fortin WJ, Crane AM. 1999. Projections of the median raphe nucleus in the rat. J Comp Neurol 407:555-82.

1059

Vertes RP, Linley SB. 2008. Efferent and afferent connections of the dorsal and median raphe nuclei in the rat. In: Serotonin and Sleep: Molecular, Functional and Clinical Aspects. Birkhäuser Basel. p 69-102.

1062 Vitalis T, Cases O, Passemard S, Callebert J, Parnavelas JG. 2007. Embryonic depletion of serotonin affects cortical development. Eur J Neurosci 26:331-344.

1064 Warembourg M, Poulain P. 1985. Localization of serotonin in the hypothalamus and the mesencephalon of the guinea-pig. Cell Tissue Res 240:711-721.

1066 Weaver KJ, Paul IA, Lin RCS, Simpson KL. 2010. Neonatal Exposure to Citalopram 1067 Selectively Alters the Expression of the Serotonin Transporter in the Hippocampus: DoseDependent Effects. Anat Rec 293:1920-1932.

Witter MP, Amaral DG. 2004. Hippocampal Formation. In: The Rat Nervous System. Elsevier. p 635-704.

1071 Xu Y, Sari Y, Zhou FC. 2004. Selective serotonin reuptake inhibitor disrupts organization of thalamocortical somatosensory barrels during development. Dev Brain Res 150:151-161.

Zeng Z, Chen TB, Miller PJ, Dean D, Tang YS, Sur C, Williams DL. 2006. The serotonin transporter in rhesus monkey brain: comparison of DASB and citalopram binding sites. Nucl Med Biol 33:555-563. 
1077 Figure Legends

1078 Figure 1: Innervation pattern of SERT-EGFP fibers across the rostro-caudal extent of 1079 thalamus.

1080 The terminal labelling density in each nucleus is written numerically within the brackets.

1081 (A-B) Sections from rostral thalamic pole (A) PVT, CM nuclei and nucleus RE (6+). PT and 1082 AV nuclei (5+). AD (4+). AM and RT* nuclei (2+). ST nuclei (3+). SM and fx (0), fibers 1083 clustered at apex of SM (arrow head). *Rostral most RT was moderately labelled (fig not 1084 shown). (B) PVT, CM, RH nuclei and nucleus RE (6+). LD and AV nuclei (5+). IAM and AD 1085 nuclei $(4+)$. AM and IAD nuclei $(2+)$. VAL and RT nuclei $(1+)$. MD and SMT nuclei (3+). 1086 MH (1+). (C-D-E) Sections from mid thalamic level (C) PVT, RH nuclei and nucleus RE (6+). 1087 IMD, LD, AV and CL nuclei (5+). CM, PCN and MD nuclei (3+), central part \& dorsal area of MD (4+). VAL, VM, RT, SMT nuclei (1+), MH (3+ on ventral area, 1+ on dorsal). (D) PVT, RH nuclei, nucleus RE and SMT (6+). IMD, CL and LD nuclei (5+). MH, LH and MD nuclei

1090 (3+), central part \& dorsal area of MD nuclei (4+). CM, PCN, PO, VAL, VPM, VPL, VM, and 1091 RT nuclei (1+) (E) PVT*, RE, SMT, LGd \& LGv nuclei (6+). LP nuclei (5+). LD nuclei (4+). $1092 \mathrm{MH}$ and $\mathrm{LH}(3+)$. MD nuclei (2+), less density on its ventral part. IMD and RH nuclei (3+). 1093 CM and RT nuclei (1+). PCN, CL, PO, VM, VAL, VPM, and VPL nuclei $(<1)$. *Ascending 1094 fiber bundles traversing ahead via PAG were clustered as bilateral bundle in PVT (arrow head).

1095 Some of them divert laterally and in-between habenula. (F-G) sections from the caudal 1096 thalamic pole F) RE and LGd \& LGv nuclei (6+), slightly less fibers on medial part of LGv. 1097 PVT nuclei (5+). LH and LP nuclei (4+). PF nuclei (1+; dorsal part, 2+). RT nuclei (1+). PO, 1098 VM, VPM and VPL nuclei (<1+). G) LGd \& LGv nuclei (6+). LP (3+) nuclei. MG nuclei (2+) 1099 with slightly higher on lateral part. PO nuclei (1+).

1100 Figure 2: Innervation pattern of SERT-EGFP fibers across the rostro-caudal extent of 1101 hypothalamus. 
(A-B) Sections from preoptic hypothalamus (A) LPO, MPO, MEPO nuclei (5+). AVPV nuclei

1103 (2+). Non-hypothalamic nuclei: SI (6+), which contains the collaterals of ascending pathway

1104 fibers detached from LPO area. MA nucleus and NDB (5+). Fibers from SI pass medially 1105 across the base of MA nucleus and NDB (yellow arrow). OT (5+). Fundus of striatum (FS)

1106 contains scattered collaterals of ascending fibers. (B) LPO area (6+). PVHap and PVpo nuclei 1107 (5+). MPN nuclei (4+). MPO area (4+) with 3+ density on its medial part. Non-hypothalamic 1108 nuclei: SI (6+). NDB (5+). MA nucleus (4+). OT (5+) with heavy clustering of fibers over its 1109 dorsal area. (C-F) Sections from supraoptic hypothalamus (C) LHA area (6+) with slightly less 1110 fibers on lateral part. AHN and ZI (5+). Fibers from LHA pass medially into the thalamus upon 1111 traversing through ZI or fx. SBPV, RCH and SO nucleus (3+). PVH; besides the part lining the 1112 ventricle (PVHpv; 5+), rest of the PVH is sparsely (1+) labelled. SCN (1+). Non-hypothalamic 1113 nuclei: SI (6+). (D) SCN; core (6+), shell (1+). Optic fibers entering into SCN were innervated 1114 by 5-HT fibers (arrow head). SBPV, PVi nuclei (5+). PVH (1+; except periventricular part, 1115 PVHpv 5+). (E) SCN (1+). SBPV area (5+). PVH nucleus (1+; except periventricular part, 1116 PVHpv 5+). (F) LHA (6+). AHN, ZI, RCH, PVH, SBPV, and PVi nuclei (5+). Fibers from

1117 LHA pass over supraoptic commissure to enter into the RCH (yellow arrow). Innervation in $1118 \mathrm{RCH}$ decreases slightly on medial parts. Dorsally, fibers from LHA enter into the RE of 1119 thalamus and into ZI. SO nucleus (3+). Arc nuclei (2+). Non-hypothalamic area: SI (6+) (G1120 H) Sections from supraoptic hypothalamus (G) LHA (6+). DMHa, TU* nuclei and PVi (5+). 1121 VMH nuclei (5+; except dm part, 2+). Arc nucleus (1+). ZI (4+). *Collaterals of pathway fibers 1122 were observed to be directly passing into TU nuclei. (H) LHA (6+). VMH, TU, PH, STN 1123 nuclei, and PVi (5+). ZI (3+). DMH nucleus (2+). Arc nucleus (1+). Some fibers from LHA 1124 enter into the rostrally located GP through cpd. (I-J-K) Sections from mamillary hypothalamus 1125 (I) LHA (6+). PH and STN nuclei (5+). PMv and PMd (4+). TMd (2+). PVp (1+). ME (0). (J) 1126 LM* nucleus, and MMme (5+). LHA, MM nuclei, TMv (4+) and PH (3+). SUM (2+). PVp 
$1127(1+) .{ }^{*}$ Collaterals of pathway fibers from VTA pass laterally to directly enter into the LM

1128 nuclei (black segmented arrow). (K) LM nucleus (5+). MM nucleus (4+) with mild (2+)

1129 innervation on medial part. SUM nucleus $(1+)$

1130 Figure 3: Innervation pattern of SERT-EGFP fibers across the rostro-caudal extent of

\section{1 amygdala.}

1132 (A-B) Rostral amygdalar section (A) MEAad, medial part of AAA (6+). Lateral part of AAA, 1133 IA, BLA and BMAa nuclei (5+). CEA, NLOT*, and COAa (4+). * Rostral NLOT (5+) (figure 1134 not shown). Non amygdalar nuclei: EPd (6+) and EPv (5+). Fibers enter into the amygdala 1135 from LHA and SI (yellow arrow). (B) *BLAa, IA (6+). MEAad, and BMAa nuclei (5+). 1136 NLOT- layer $1 \& 3(4+)$, layer $2(1+)$. CEA and BA nuclei (3+). COAa (2+). Non amygdalar 1137 nuclei: EPd (6+) EPv (5+). * BLAa was bounded by very dense clustered fibers within the 1138 external capsule on medial and ventral side. (C-D) Mid amygdalar section (C) BLAa (6+). 1139 MEAad, and IA nuclei (5+). LA, CEA, MEAav, BMAp, COApl, and PAA (3+). Fibers 1140 travelling within stria terminalis (st) project into the CEA and MEAad nuclei. (D) BLAa and 1141 BLAp (6+). BLAv nucleus (5+). BMAp nucleus (4+), shows the intranuclear gradient. LA, 1142 CEA, MEApd, MEAav, COApm, COApl, and PAA (3+). Fibers travelling within stria 1143 terminalis (st) project into the CEA and MEApd nuclei. (E-F) Caudal amygdalar section (E) 1144 BLAp nuclei (6+). BLAa, BLAv, BMAp, COA, and PAA (5+). MEApd, MEApv, and LA 1145 nuclei $(3+)$. Stria terminalis (st) was very densely $(6+)$ clustered with SERT-EGFP fibers both 1146 in (E) and (F). (F) BLAp nuclei (6+). PA, BLAv, COA (pm,pl), and PAA (5+). BMAp nucleus

1147 (3+). Transition zone (TR) between PAA and PIR was mildly (2+) innervated.

1148 Figure 4: Innervation pattern of SERT-EGFP fibers across the rostro-caudal extent of 1149 septal nuclei.

1150 (A-B) Sections from rostral septal level (A) LSr nuclei (6+), collaterals of pathway fibers 1151 travelled through LSr to form the supracallosal bundle ( $\mathrm{scb}$ ) located above the CC (arrow head). 
1152 NDB (4+). SH nuclei (2+). IG contains the collaterals of pathway fibers which innervate the

1153 medial cortex. Non-septal nuclei: TTd (3+) (B) NDB, MS, and LSr nuclei (5+). Ventrodorsally

1154 running collaterals of pathway fibers were clustered very densely $(6+)$ in the medial and lateral

1155 border of MS which terminated by bounding the SH nuclei dorsally. SH nuclei (1+). (C-D)

1156 Sections from mid septal level. LSr nuclei (5+). NDB and medial part of MS (3+). Fibers can

1157 be observed entering into the septum via SI. Ventrodorsally running collaterals of pathway

1158 fibers clustered very densely (6+) in the lateral border of MS which reached upto the ventral

1159 border of CC. (C) LSc (3+) with vertically oriented fibers abutting the CC (arrow head). Islm

1160 (0) (D) LSc (2+) with vertical fibers abutting CC (black arrow head). Very dense (6+) cluster

1161 of fibers between LSr and NAc (yellow arrow head). Islm (5+). (E-F) Sections from caudal

1162 septal level. (E) Medially diverted collaterals of ascending fibers from LPO nuclei entered into

1163 the septum. LSr nuclei (4+). Mediolaterally, directed fibers in LSr. LSv nuclei (3+), except

1164 densely clustered fibers at its dorsal part (arrow). LSc $(1+)$ with vertical fibers at the dorsal

1165 border. Column of fornix ( $\mathrm{fx})(0)$. Thin fascicle of fiber passing ventrodorsally in between the

1166 two columns. (F) SF nuclei (4+); mediolaterally oriented fibers. LSc nuclei (1+) with vertical

1167 fibers at its dorsal border (arrow head). TRS (1+). LSv nucleus (3+).

1168 Figure 5: Innervation pattern of SERT-EGFP fibers across the rostro-caudal extent of

1169 caudoputamen and nucleus accumbens.

1170 (The images are the serial sections arranged rostro-caudally) (A) CP (2+). NAc (4+). Fibers

1171 entered into the $\mathrm{CP}$ and NAc through collaterals of fibers running in external capsule (arrow

1172 head) (A-C). (B) CP (3+ on lateral area and 2+ on medio-dorsal part). Fibers running within

1173 external capsule (arrow head) wind up around olfactory limb of anterior commissure (aco) or

1174 directly enter into the CP and NAc (3+). (C) collaterals of pathway fibers within SI wind around

1175 aco to enter into the medial side of CP (segmented black arrow line). Medial part (3+) have

1176 higher innervation compared to lateral $(2+)$. Region of thalamocortical fibers traversing 
1177 through CP appear as circular gap (green arrow head). Collaterals of ascending pathway fibers

1178 appear as patch in CP (red arrow head) and NAc (blue arrow head). NAc $(2+)$, in the area

1179 except the patch (blue arrow head) (D) CP (3+ homogeneous distribution throughout). GPe

$1180(6+)$. Empty holes in the $\mathrm{CP}$ and GPe are areas of non-labelled thalamocortical fibers.

1181 Collaterals of pathway fibers in the SI, directly innervated the GPe. (E) CP (4+). GPe (6+).

1182 Fibers arising from main ascending bundle at LHA entering into the GPe through SI. Fibers

1183 were very densely clustered only around the thalamocortical fibers (whorl like pattern) in the

1184 GPi and sparse elsewhere. (F) Sagittal section showing both caudoputamen and nucleus

1185 accumbens. Gap indicates unlabeled thalamocortical fibers. Thin, relatively straight fibers were

1186 seen terminating mainly in the NAc (red arrow head) and few in the CP. Some of them entered

1187 into the external capsule (yellow arrow head) which provided the innervation both to the cortex

1188 and CP. (G) enlarged view of nucleus NAc showing the straight, non-varicose terminal fibers

1189 seen in E (red arrow head)

1190 Figure 6: Innervation pattern of SERT-EGFP fibers across the rostro-caudal extent of

1191 bed nuclei of stria terminalis and olfactory tubercle.

1192 (The images are the serial sections arranged rostro-caudally) A-B: anterior BNST. Fibers

1193 entered into the BNST via collaterals of pathway fibers clustered within SI. (A) am and al

1194 nuclei (4+). (B) am and al nuclei (5+). ov and fu nuclei (2+). C-D: posterior BNST. (C) am and

1195 al nuclei (6+). dm, mg, v, and rh nuclei (5+). Pr nuclei (2+), except at ventral area. Fibers on

$1196 \mathrm{pr}$, am, al and rh nuclei entered into the stria terminalis (st). (D) tr nuclei (6+). if, v, al nuclei

$1197(5+)$. pr nuclei $(2+)$. Fibers from BNST entered into the stria terminalis (st) to run dorsally

1198 above the thalamus. (A-B) OT (6+). Fibers in OT were clustered around isl; while latter were

1199 almost devoid of labelling. (C-D) OT (5+); fibers distributed were fine in morphology and

1200 homogeneously distributed throughout. 
Figure 7: Innervation pattern of SERT-EGFP fibers across the rostro-caudal extent of

\section{hippocampus}

1203 (A) Rostral end of the dorsal hippocampus. Fibers entered into the hippocampus through dorsal

1204 fornix (df) and fimbria (fi). Some of these collaterals passed into the alveus (alv) and ramified 1205 into the SO. SO and SR (4+). SP, PO and MO layer (1+). SG (0). (B-C) SO and SR of CA1 and CA2 zone of HPF (3+). SO and SR of CA3 zone of HPF (4+). Whole extent of SLM (5+). Whole extent of SP (1+). Dorsal part of MO layer of DG (3+). Ventral part of MO layer of DG and PO (1+). SG (0). Fibers enter into the hippocampus through fimbria (fi) and fibers spreading laterally from SLM made the CA3 SO and CA3 SR appear more clustered with fibers respectively. (C) Retrosplenial part of dorsal hypothalamus. Collaterals of pathway fiber from supracallosal bundle (scb) passed ventrally into the hippocampus (black arrow) mainly into the alvelus (alv) and SLM. (D)* Ventral hippocampus. SO, SR of CA1 (3+). SLM (6+). MO layer $(3+)$ except at apex. SR of CA3 (5+). SO of CA3 (3+). SP and PO (1+). SG (0). SUBv and SUBd (3+). Fibers passing through the SLM moved out laterally to innervate the rhinal cortex. (E) Image showing the sources of innervation fibers of the hippocampus. supracallosal bundle (scb) running dorsally above corpus callosum (CC) wind around the splenium to enter the

1217 hippocampus (yellow arrow head). Collaterals of pathway fibers enter into the septal nuclei run

1218 dorso-caudally to pass into the dorsal fornix (df) and fimbria (black arrow) which subsequently 1219 innervate the hippocampus.

*(D) Image showing both the ventral hippocampus and cortical section from occipital pole.

1221 RSP, Visual and auditory cortex: layer $1(4+)$, layer $2 / 3$ and $4(1+)$, underlying layers (2+). Temporal association area (TEa) (3+). Rhinal cortex: $6+$ except in second and deepest layer. 
1225 (The images are serial cortical sections arranged rostro-caudally) (A) Rostral pole of the

1226 cortex. Layer 1 in all the areas (5+). PL area: patch like cluster of fibers. layer 2 and 2/3 (4+).

1227 rest of the layers (3+). ACAd area (3+). MOs area: layer (5+) and deeper part of $6 \mathrm{a}(4+)$, other 1228 layers (3+). MOp area and AId area: layer $5(5+)$, rest of the layers (3+). ORB area: layer 5 $1229(4+)$ and rest of the layers (3+) (B) Layer 1 in all areas (5+). ILA, PL, and ACAd area (3+). 1230 Fibers from endopiriform nuclei (EPd) enter into the medial cortex through the deeper layer of 1231 ILA and PL cortex. MOs area: $3+$ in rest of the layers except $6 \mathrm{~b}(2+)$. MOp area: Layer 5 (4+). 1232 Rest of the layers (3+). SSp, GU and AI area: layer 5 and $6 \mathrm{~b}(5+)$. Layer $6 \mathrm{a}(3+)$. Layer 2/3 $1233(2+)$. ORB area: $2+$ in rest of the layers except layer $5(3+)$. Fibers enter into the layer 1 of 1234 lateral cortex through areas around rhinal fissure. (C) Layer 1 in all areas (5+). ILA, PL and $1235 A C A d$ area $(3+)$ in rest of the layers. Fibers enter into this medial cortex via medial forebrain bundle (mfb) (black arrow line) and indusium griseum (ig) (yellow arrow head). MOs and Mop

1237 area: layer 5 and $6 \mathrm{a}(3+) .2+$ in rest of the layers. SSp area: Layer 5 and $6 \mathrm{~b}(5+)$. Fibers 1238 clustered within the claustrum (CLA) distribute to layer 6a (3+). Layer 2/3 and 4 (2+). GU and 1239 AI area: layer $5(4+)$. Rest of the layers (3+). Fibers clustered within EPd enter into the lateral 1240 cortex mainly through the layer 1 and Layer 5. (D) Layer 1 in all areas (5+). $A C A v$ and $A C A d$ $1241(2+)$ except at the deeper layers where fibers from supracallosal bundle (scb, arrow head) pass 1242 dorsally towards the motor areas and horizontally into layer 6b. MOs and MOp area (3+) in 1243 upper layers; $2+$ in deeper layers 6a and 6b. SS area: layer 5 (5+). Layer 6a and 6b in the barrel 1244 field (bf) area (4+). Rest of layer 6 including layer 2/3 (2+). Barrel field area in the SS cortex 1245 receive higher projections compared to rest of the layers. Visceral area (VISC), GU and AIp 1246 area: Fibers from EPd traverse into the lateral cortex through the layer 1, 5 and $6 \mathrm{~b}(5+$ in all 1247 of these layers) of these areas. Fibers travelling through external capsule (ec, arrow head) also 1248 provide innervation to deeper cortical layers and caudoputamen. Piriform cortex (PIR): $3+$ in 1249 all of the layers without laminar pattern. (E) Layer 1 in all areas (5+). RSP area (3+). A vertical 
band of fibers between layer 2 and 2/3. Fibers from supracallosal bundle (scb, arrow head) pass dorsally towards the motor areas. Motor areas (MOs and MOp): layer $5(4+)$ and rest of the layers (3+). Somatosensory area (SSs and SSp): layer 5 (4+) and rest of the layers (2+). (F) Layer 1 in all areas (5+). Retrosplenial area: RSPagl (3+). RSPv and RSPd (2+). Visual area: deeper layers $(6 a, 6 b)(3+)$. Upper layers $(1+)$. Auditory area: layer 5 and below (4+). Layer 2/3 and $4(1+)$. Rhinal and temporal association area (TEa): $5+$ in rest of the layers except layer 2/3 (2+). Piriform cortex (PIR): laminar pattern, layer $1(5+)$, deeper layers $(3+)$. (G) Layer 1 in all areas (5+). Retrosplenial cortex (RSP) (2+). Visual and auditory area (1+). Rhinal and temporal association area (TEa): $5+$ in rest of the layer except layer 2/3. (H) Sagittal cortical section showing the dense cluster of collateral pathway fibers in the induseum griseum (IG) (arrow head) which is one of the main sources of innervation to the medial cortex.

Figure 9: Innervation pattern of SERT-EGFP fibers in the olfactory bulb.

(A) SERT-EGFP fibers arrangement in the main olfactory bulb (MOB) exhibit the laminar pattern. GL (5+). OPL (1+). MI, IPL and GR layer (4+). OV (1+). Optic nerve layer (onl) (0). (B) Accessory olfactory bulb (AOB). GR layer (1+). MI and GL layer (0). Anterior olfactory nuclei (AON) acted as the route for passage for the collaterals of ascending fibers reaching to MOB

\section{Figure 10. Innervation pattern of SERT-EGFP fibers in the cerebellum.}

1268 Sparsely (1+) labelled layers of the cerebellum. Outermost molecular layer (MO) and white 1269 matter (WM) tree almost appear scanty. Fibers are mainly distributed in the pyramidal (py, 1270 arrow head) or granular (gr) layer. Moderate (3+) labelling within the deep cerebellar nuclei 1271 (IP and FN). Fibers from raphe nuclei passed dorsally directly into the deep nuclei.

Table 2 Innervation density across different brain areas and their sub-divisions

\begin{tabular}{ll}
\hline Brain areas and their subdivisions & Innervation density change rostro-caudally \\
\hline THALAMUS & \\
Midline nuclei & $6+$ to $5+$ \\
Paraventricular Thalamic nucleus (PVT) &
\end{tabular}


Paratenial nucleus (PT)

Rhomboid nucleus (RH)

Nucleus reuniens (RE)

Intralaminar nuclei

Central lateral nucleus (CL)

Paracentral nucleus (PC)

Central medial nucleus (CM)

Parafascicular nucleus (PF)

\section{Association nuclei}

Mediodorsal nucleus (MD)

Intermediodorsal nucleus (IMD)

Submedial thalamic nucleus (SMT)

Anterodorsal nucleus (AD),

Anteroventral nucleus (AV)

Anteromedial nucleus (AM)

Interanteromedial nucleus (IAM)

Lateral dorsal nucleus (LD)

Lateral posterior nucleus (LP)

Inter anterodorsal nucleus (IAD)

\section{Principal nuclei}

Ventral posteromedial nucleus (VPM)

Ventral posterolateral nucleus (VPL)

Ventroanterior lateral complex (VAL)

Ventromedial nucleus (VM)

Posterior nucleus (PO)

Lateral geniculate nucleus (LG)

Medial geniculate nucleus (MG)

\section{Epithalamus}

Medial habenula

Lateral habenula

\section{Reticular nucleus}

\section{Other areas}

fasciculus retroflexus (fr)

mamillotegmental tract (mmt)

posterior commissure (pc)

internal capsule (ic)

cerebral peduncle (cpd)

Internal capsule (int)

stria medullaris $(\mathrm{sm})$

stria terminalis (st)

\section{HYPOTHALAMUS}

Preoptic region

Median preoptic nucleus (MEPO)

Medial preoptic area (MPO)

Anteroventral periventricular nuclei (AVPV)

Medial preoptic nucleus (MPN)

Lateral Preoptic nucleus (LPO)

\section{Supraoptic region}

Supraoptic nucleus (SO)

Retrochiasmatic nucleus ( $\mathrm{RCN})$

Anterior hypothalamic nucleus (AHN)

Suprachiasmatic nucleus (SCN)

Subparaventricular zone (SBPV)

Lateral hypothalamic area (LHA)

Paraventricular hypothalamic nucleus (PVH)
$5+$

$6+$ to $3+$

$6+$

$5+$ to $1+$

$3+$ to 0

$6+$ to $1+$ to $2+$

$2+($ dorsal $), 1+($ ventral $)$ (intranuclear gradient)

$3+$ to $2+(4+$ on dorsal and central part $)$

$5+$ to $3+$

$3+$ to $1+$ to $6+$

$4+$

$5+$

$2+$

$4+$ to $2+$

$5+$ to $4+$

$5+$ to $4+$

$2+$

$<1+$

$<1+$

$<1+$

$<1+$

$1+$ to $<1$

$6+$

$2+$

$1+$ to $3+$ (intranuclear gradient)

$3+$ to $4+$

$3+$ to $1+$

0

0

0

0

0 (fibers traversing through at some level)

0

0 (fibers clustered at dorsal part)

$(3+)$ (fibers passing through)

$5+$

$5+$ to $4+(3+$ on medial part $)$

$2+$

$4+$

Route for the ascending forebrain bundle

$3+$

$4+$ to $5+$

$5+$

$1+$ to $6+$ (intranuclear gradient at midlevel)

$3+$ to $5+$

Route for the ascending forebrain bundle 
Periventricular part (PVHpv)

Rest of other areas

Tuberal region

Ventromedial hypothalamic nucleus $(\mathrm{VMH}) \quad 5+($ dorsomedial part 2+)

(except dorsomedial part)

Dorsomedial hypothalamic nucleus (DMH)

Arcuate hypothalamic nucleus (ARH)

Tuberal nucleus (TU)

\section{Mamillary region}

Posterior Hypothalamic nucleus (PH)

Posterior part of periventricular nucleus $(\mathrm{PVp})$

Premamillary mamillary nucleus, ventral $(\mathrm{PMv})$

$\&$ dorsal (PMd)

Lateral mamillary nucleus (LM)

Tuberomammillary mamillary nucleus, dorsal

(TMd) \& ventral (TMv)

Medial mamillary nucleus (MM)

Median part of MM (MMme)

Supramamillary nucleus (SUM)

Median eminence (ME)

\section{Zona incerta (ZI)}

Subthalamic nucleus

Periventricular nucleus (PV); preoptic (Pvpo), 5+ anterior (Pva) and intermediate (Pvi) part

Other areas

Optic tract (opt)

Fornix (fx)

Fasciculus retroflexus (fr)

Mamillotegmental tract (mtt)

Cerebral peduncle (cpd)

Anterior commissure (aco)

Substantia Innominata (SI)

Magnocellular Nuclei (MA)

Nucleus of diagonal band (NDB)

\section{AMYGDALA}

\section{Deep or basolateral group}

Lateral amygdalar nucleus (LA)

Basolateral amygdalar nucleus (BLA)

Anterior (BLAa)

Posterior (BLAp)

Ventral part (BLAv)

Basomedial amygdalar nucleus (BMA)

Anterior part (BMAa)

Posterior part (BMAp)

Superficial or cortical-like group

Nucleus of lateral olfactory tract (NLOT)

Bed nucleus of accessory olfactory tract (BA)

Cortical amygdalar nucleus

$$
\text { Anterior (CoAa) }
$$

Posterior (CoAp)

Piriform-amygdaloid area (PAA)

\section{Centromedial group}

Medial amygdalar nuclei (MeA) Anterodorsal part (MEAad)

Anteroventral part (MEAav)

$$
\begin{aligned}
& 5+ \\
& 1+\text { to } 5+
\end{aligned}
$$

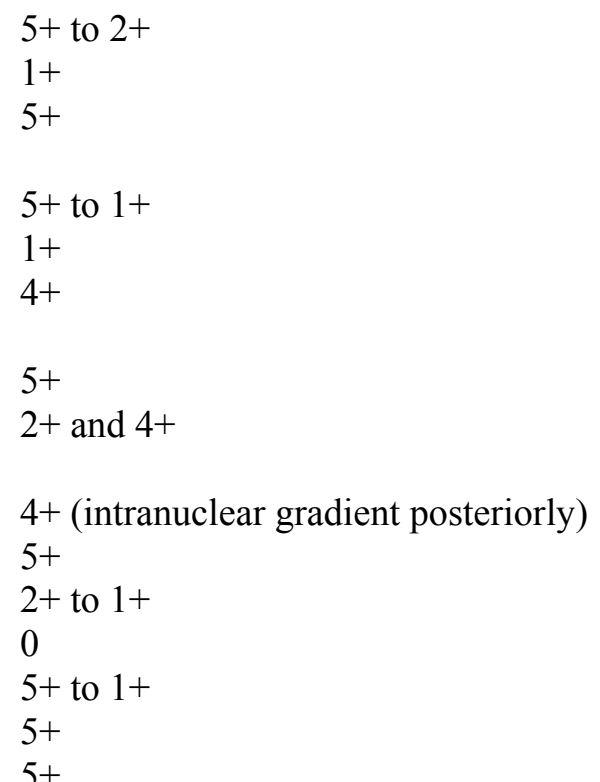

$4+$ (intranuclear gradient posteriorly)

$5+$

$2+$ to $1+$

$5+$ to $1+$

$5+$

0

0

0

0

0 (at some level fibers traverse through it)

0

$6+$

$5+$ to $4+$ (heavy fibers at its ventral part)

$5+$

$3+$

$6+$ to $5+$

$6+$

$5+$

$5+$

$3+$ to $5+$ to $3+$

$5+$ to $4+$ (laminar)

$3+$

$2+$ to $5+$

$4+$ to $2+$

$3+$ to $5+$

$3+$ to $5+$

$6+$ to $5+$

$3+$ 
Posteroventral part (MEApv)

Posterodorsal part (MEApd)

Central amygdalar nuclei (CeA)

\section{Other nuclei}

Anterior amygdalar area (AAA)

Intercalated cell masses (IC)

Posterior amygdaloid nucleus (PA)

Non amygdalar nuclei

Endopiriform nuclei; dorsal (EPd) and ventral (EPv)
$3+$

$3+$

$4+$ to $3+$

$5+(6+$ on its medial parts $)$

$6+$ to $5+$

$5+$

$6+$ and 5+ respectively

$5+$ to $3+$

$5+$ to $3+(6+$ at its limiting zone with LS $)$

$3+$ to $1+$

$6+$ to $5+$ to $4+$

$3+$ (except the dorsal edge)

$1+$

0 to $5+$

$1+$

$4+$

Column of fornix (fr) 0

Anterior commissure, olfactory (aco) and 0 temporal (act) limb

BASAL GANGLIA

Caudoputamen (CP)

Globus pallidus (GP)

$2+$ to $3+$ to $4+$

$6+$ (whorl in internal segment)

$4+$ to $3+$

0

Corpus callosum (CC)

BED NUCLEI OF STRIA TERMINALIS

Anterior division of BNST

Anteromedial (Am) area

Anterolateral $(\mathrm{Al})$ area

Oval nucleus (Ov)

Fusiform nucleus ( $\mathrm{Fu}$ )

Posterior division of BNST

Principal nucleus (Pr)

Dorsomedial nucleus (Dm)

Rhomboid nucleus (Rh)

Magnocellular nucleus (mg)

Ventral nucleus (V)

$4+$ to $5+$

$4+$ to $5+$

$2+$

$2+$

$2+$ (fibers clustered at ventral area)

$5+$

$5+$

$5+$

$5+$

$5+$

$6+$

$6+$

\section{HIPPOCAMPUS}

Hippocampal formation (HPF)

stratum oriens (SO)

pyramidal layer (Py)

stratum radiatum (SR)

stratum lacunosum molecularae (SLM)

Dentate gyrus (DG)

molecular layer (Mo)

granule cell layer (SG)
3+ (CA3 SO: $4+)$

$1+$

3+ (CA3 SR: $4+)$ (CA3 SR of ventral HPF: $5+)$

$5+$ (slm of ventral hippocampus: $6+$ )

$3+$ (dorsal layer), $2+$ (ventral layer $)$

0 
polymorph layer $(\mathrm{Po})$

Subiculum (SUB) 3+

\section{CORTEX}

Prefrontal cortex

Prelimbic area (PL)

Infralimbic area (IL)

Anterior cingulate area (ACA)

Agranular insular cortex (AI)
Patch like fiber cluster in upper layers in 4+ density (rostral pole) to $3+$ density (caudal pole); except $5+$ density in layer 1

$3+$ density rostro-caudally, except $5+$ density in layer 1

$3+$ density rostro-caudally, except $5+$ density in layer 1

$5+$ density in layer 1 and 5 (rostral pole) to $5+$ density in layer 1,5 and $6 \mathrm{~b}$ (caudal pole)

$3+$ density (rostral pole) slight decrease in density caudally

$5+$ density in layer 1

$5+$ to $4+$ density change in layer 5 rostrocaudally

$3+$ density in other layers

Somatosensory cortex

$5+$ density in layer 1

$5+$ to $4+$ density change in layer 5

$3+$ to $2+$ density change in other layers

Barrel field area

$5+$ density except at layer $2 / 3$

Auditory and Visual cortices

$5+$ to $4+$ density change in layer 1
$1+$ density in layer $2 / 3$ and 4

$3+$ to $2+$ density change in other layers

\begin{tabular}{ll}
\hline Rhinal area & $2+$ density in layer $2 / 3$ (rostro-caudally) and the \\
& deepest layer of caudal rhinal area \\
& $5+$ to $6+$ density change in other layers \\
\hline Piriform cortex & $3+$ density at rostral pole (no laminar pattern) \\
& $5+$ density at layer 1 and $3+$ in underlying layers \\
at caudal pole
\end{tabular}

\section{OLFACTORY BULB}

Main Olfactory Bulb (MOB)

Glomerular layer (Gl)

Outer plexiform layer (OPL)

Mitral layer (Mi)

Internal plexiform layer (IPL)

Granule layer $(\mathrm{Gr})$

Olfactory ventricle (OV)

Olfactory nerve layer (ONL)

Accessory Olfactory Bulb (AOB)

Glomerular layer (gr) 0

Mitral layer (MI) 0

Granule layer (gr) $1+$

Anterior olfactory nuclei (AON) $5+$

Olfactory tubercle (OT) $5+$

Island of Calleja (isl) 0

\section{CEREBELLUM}

Purkinje cell layer and granular cell layer

$1+$

Molecular cell layer and white matter

0

Deep cerebellar nuclei 


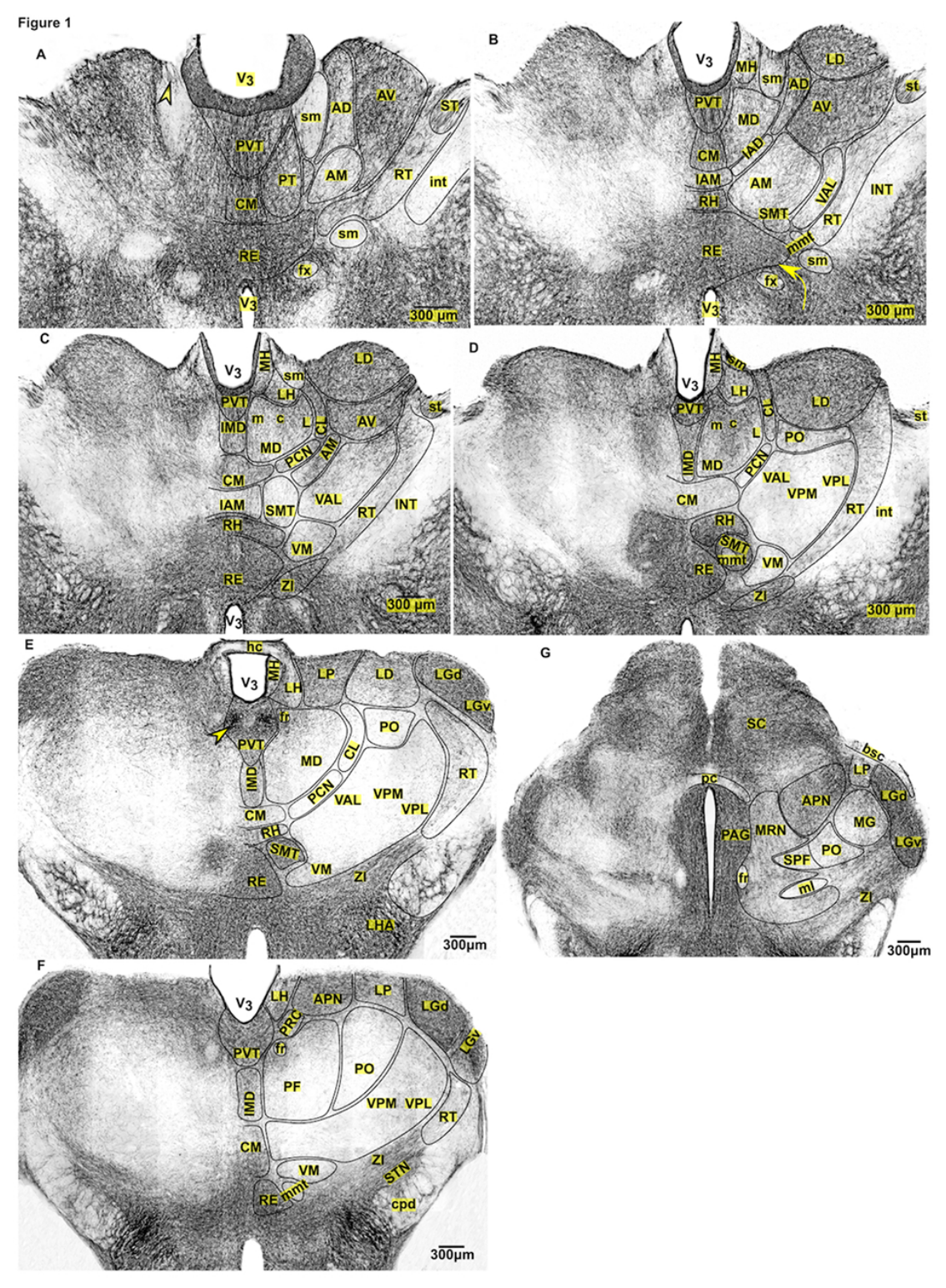

Fig. 1 


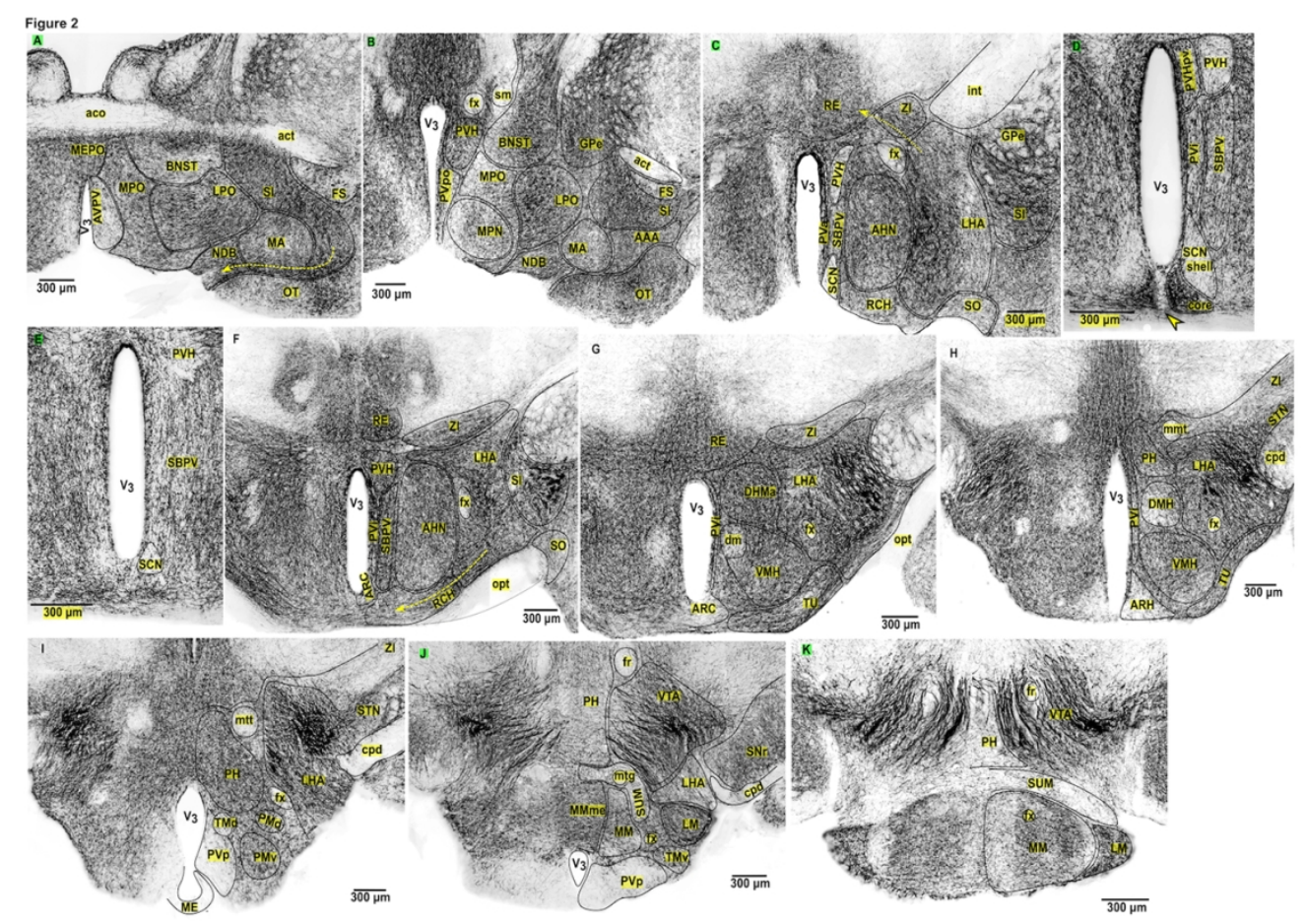

Fig. 2 


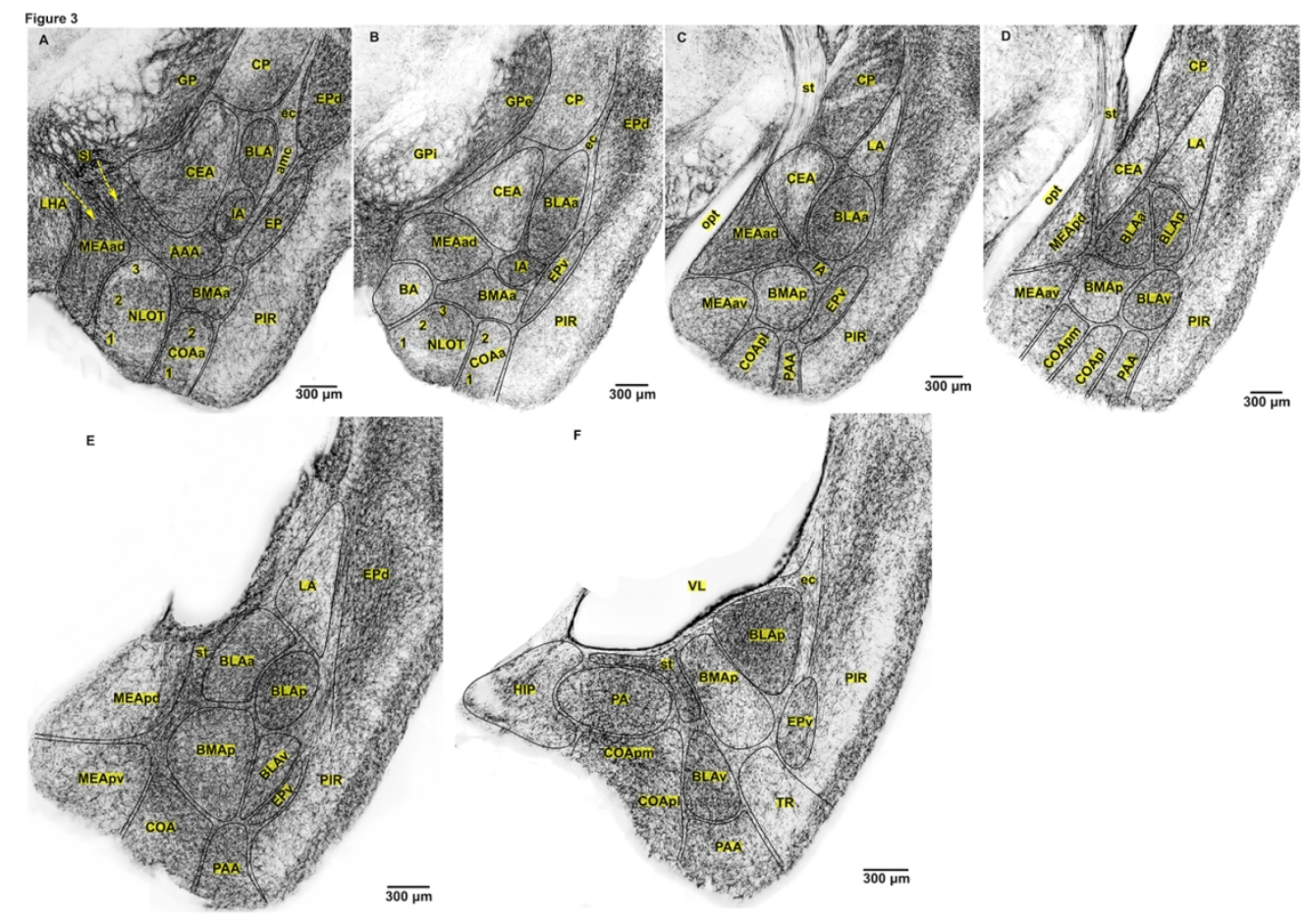

Fig. 3 


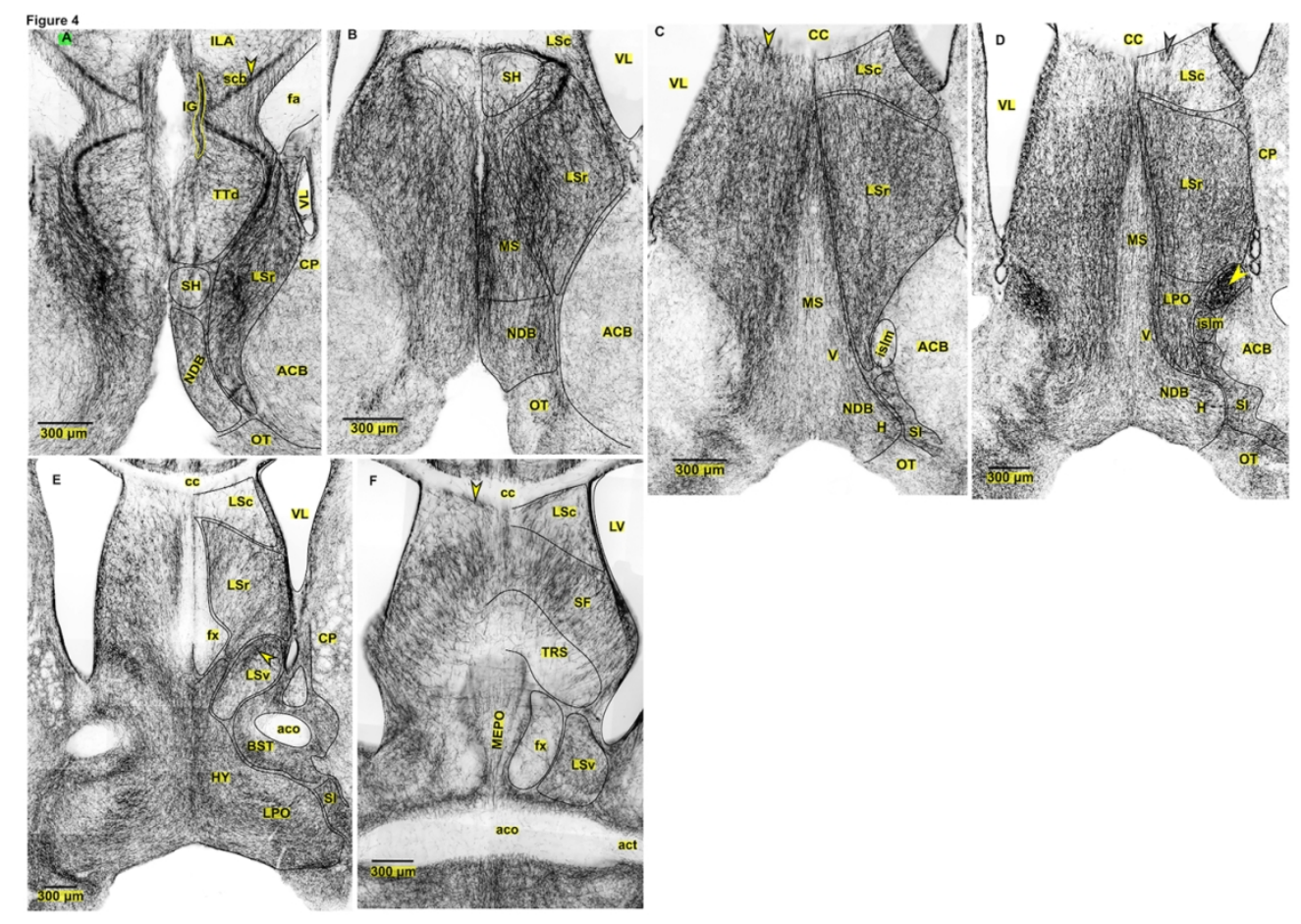

Fig. 4 


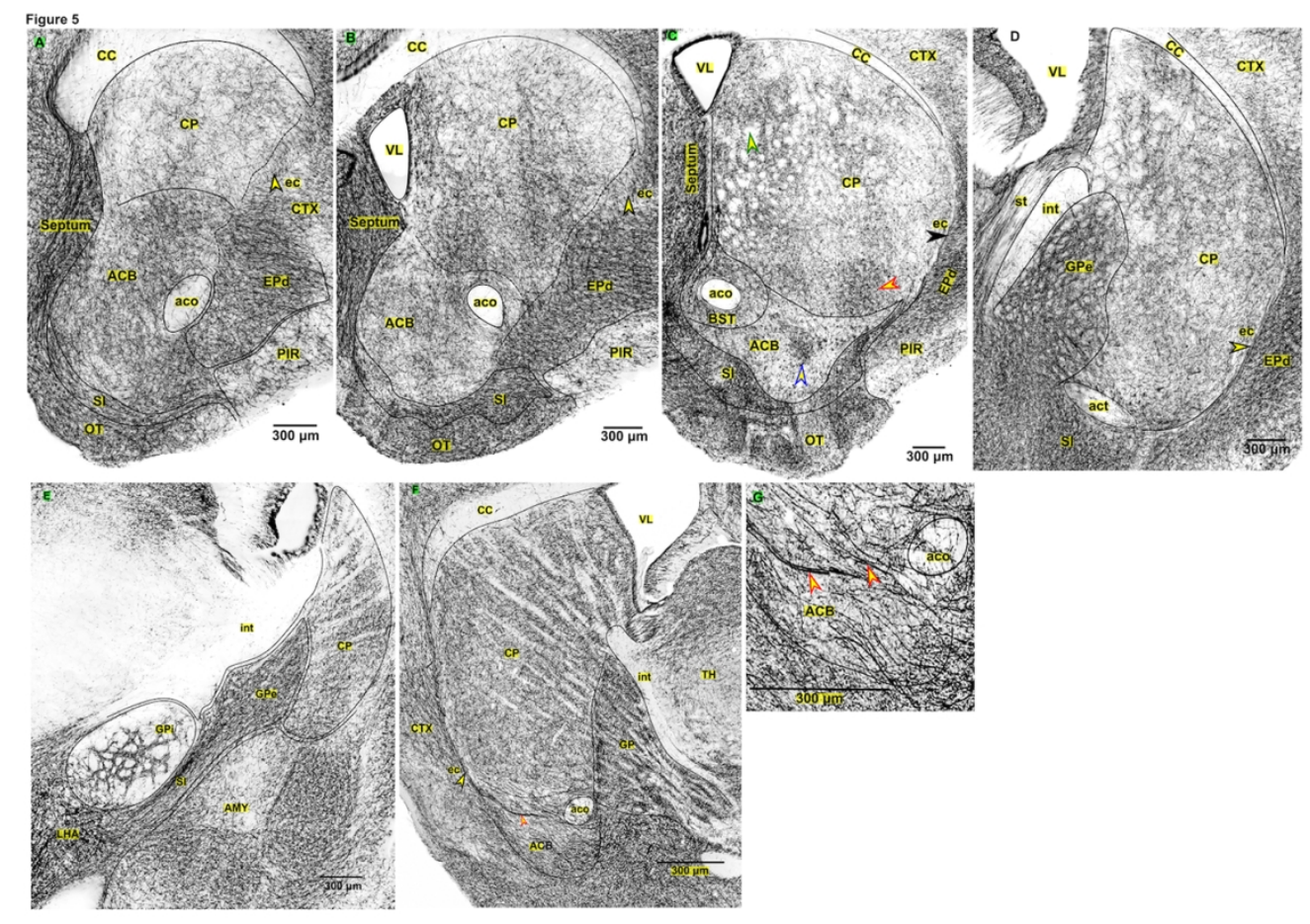

Fig. 5 


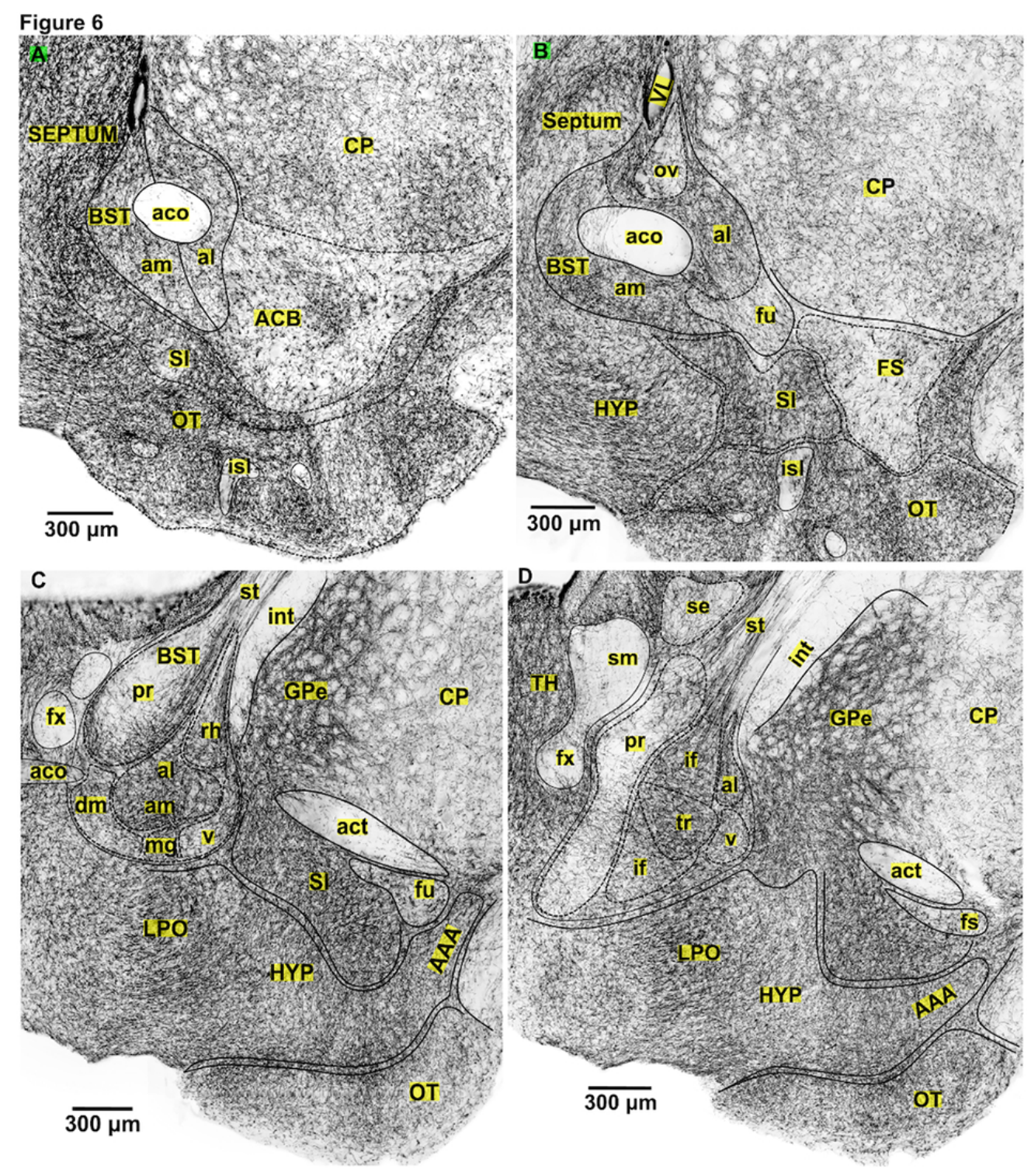

Fig. 6 


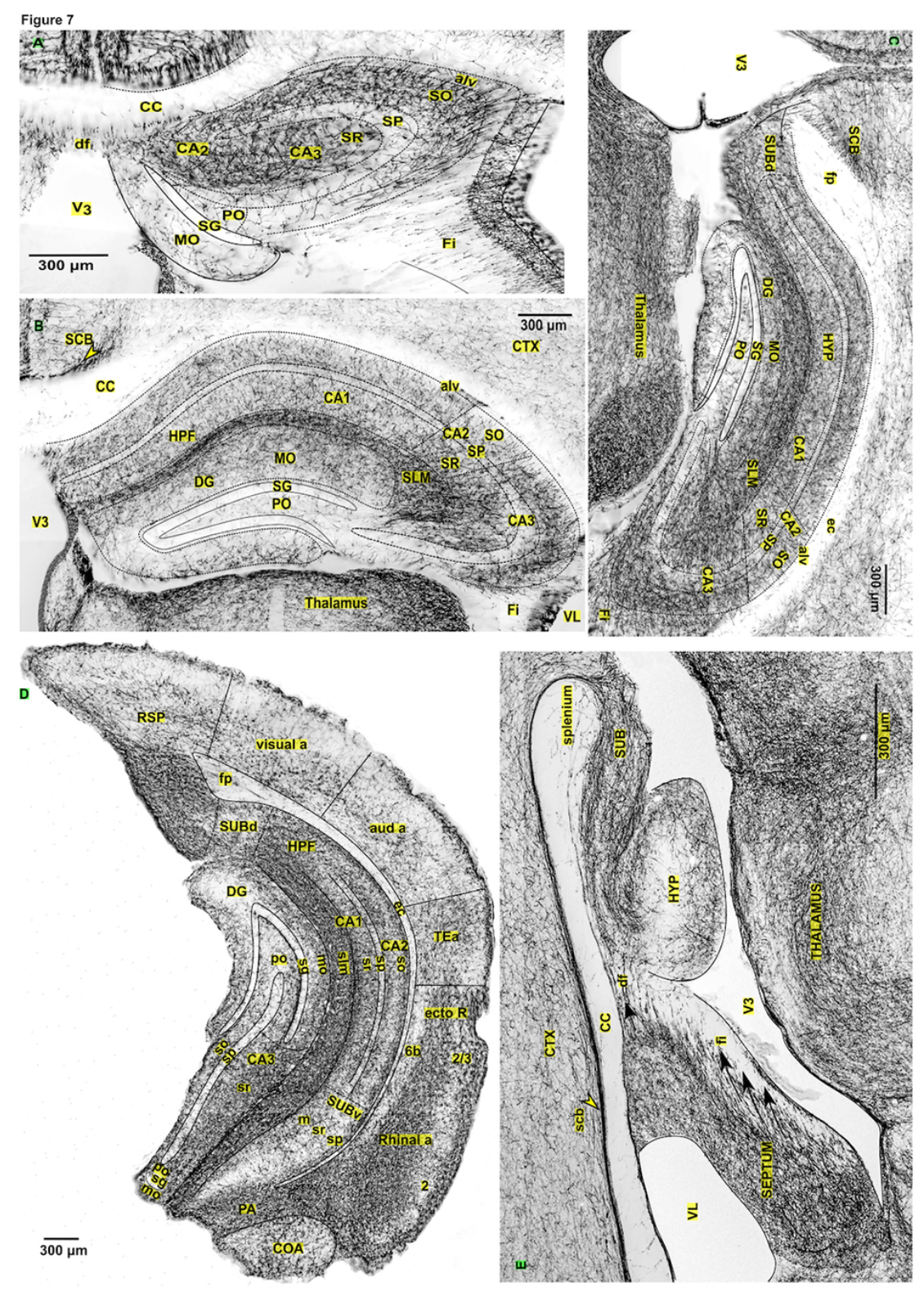

Fig. 7 


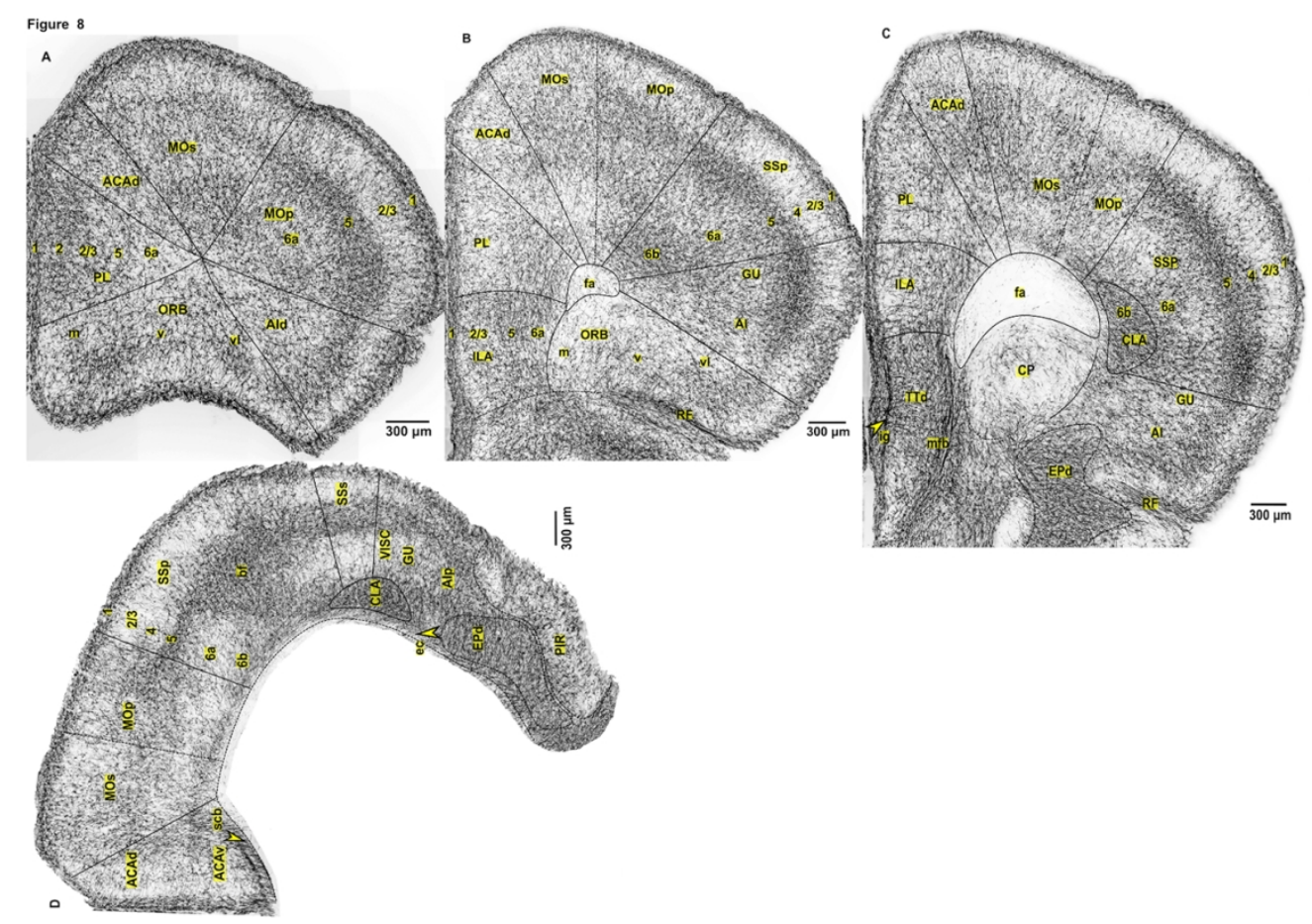

Fig. 8 

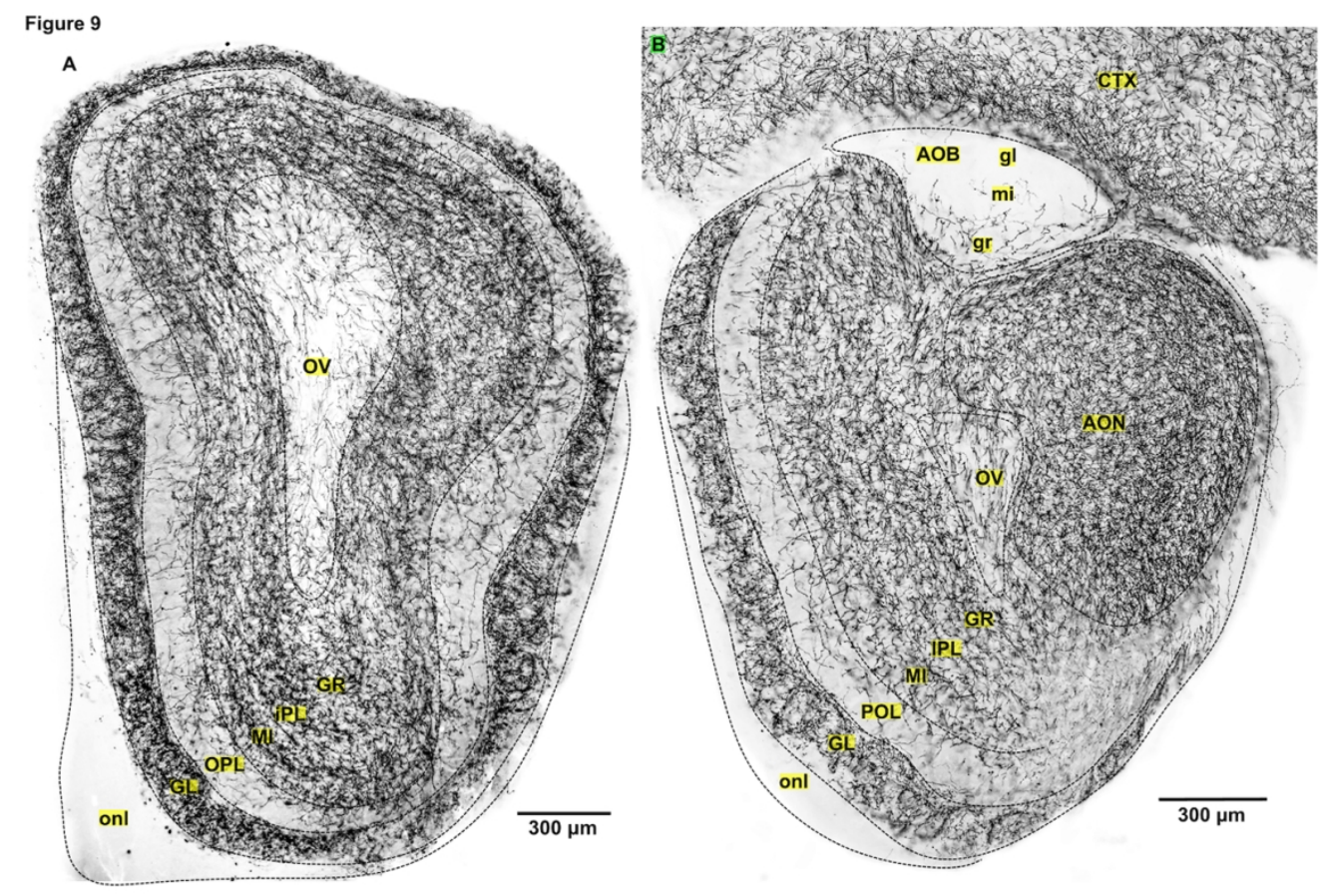

Fig. 9 


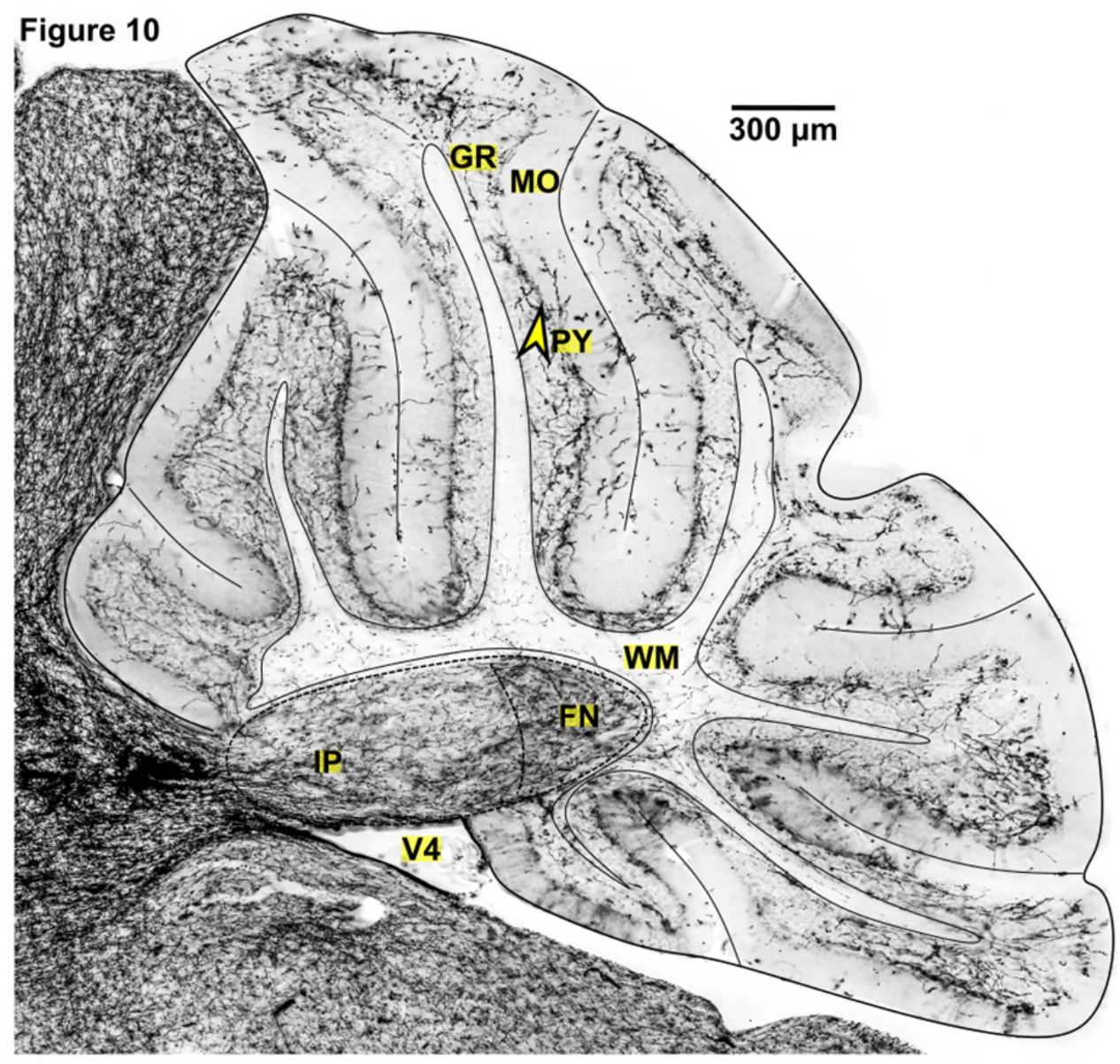

Fig. 10

$80 \times 72 \mathrm{~mm}(300 \times 300$ DPI $)$ 